\title{
UNCONDITIONAL STABILITY AND CONVERGENCE OF FULLY DISCRETE SCHEMES FOR $2 D$ VISCOUS FLUIDS MODELS WITH MASS DIFFUSION
}

\author{
FRANCISCO GUILLÉN-GONZÁLEZ AND JUAN VICENTE GUTIÉRREZ-SANTACREU
}

\begin{abstract}
In this work we develop fully discrete (in time and space) numerical schemes for two-dimensional incompressible fluids with mass diffusion, also so-called Kazhikhov-Smagulov models. We propose at most $H^{1}$-conformed finite elements (only globally continuous functions) to approximate all unknowns (velocity, pressure and density), although the limit density (solution of continuous problem) will have $H^{2}$ regularity. A backward Euler in time scheme is considered decoupling the computation of the density from the velocity and pressure.

Unconditional stability of the schemes and convergence towards the (unique) global in time weak solution of the models is proved. Since a discrete maximum principle cannot be ensured, we must use a different interpolation inequality to obtain the strong estimates for the discrete density, from the used one in the continuous case. This inequality is a discrete version of the GagliardoNirenberg interpolation inequality in $2 D$ domains. Moreover, the discrete density is truncated in some adequate terms of the velocity-pressure problem.
\end{abstract}

\section{INTRODUCTION}

1.1. The models. Let $\Omega \subseteq \mathbb{R}^{2}$ be a bounded domain with boundary $\Gamma$ that is regular enough, and $[0, T](T>0)$ the time interval of observation. We will use the notation $Q=\Omega \times(0, T), \Sigma=\Gamma \times(0, T)$.

We are going to study two models, which can be deduced from the following compressible Navier-Stokes system in $Q$ :

$$
\left\{\begin{array}{l}
(\rho \boldsymbol{v})_{t}+\nabla \cdot(\rho \boldsymbol{v} \otimes \boldsymbol{v})-\mu \nabla \cdot(\Psi(\rho) \nabla \boldsymbol{v})-(\mu+\tilde{\lambda}) \nabla(\nabla \cdot \boldsymbol{v})+\nabla q=\rho \boldsymbol{f}, \\
\rho_{t}+\nabla \cdot(\rho \boldsymbol{v})=0
\end{array}\right.
$$

where $\boldsymbol{v}: Q \rightarrow \mathbb{R}^{2}$ is the (compressible) velocity field, $q: Q \rightarrow \mathbb{R}$ is the pressure and $\rho: Q \rightarrow \mathbb{R}$ is the density of the fluid. Moreover, $f: Q \rightarrow \mathbb{R}^{2}$ is the external force, $\mu$ and $\tilde{\lambda}$ are viscosity coefficients which are assumed to be constant and such that $\mu>0$ and $3 \tilde{\lambda}+2 \mu>0$ (hypothesis known as the Bulk viscosity) and $\Psi: \mathbb{R} \rightarrow \mathbb{R}_{+}$ is a given positive function.

Received by the editor August 24, 2006 and, in revised form, July 10, 2007.

2000 Mathematics Subject Classification. Primary 35Q35, 65M12, 65M60.

Key words and phrases. Kazhikhov-Smagulov models, finite elements, stability, convergence.

The first author was supported in part by the Spanish projects BFM2003-06446-C02-01 and PHB2005-0042-PC.

The second author was supported by the Spanish projects BFM2003-06446-C02-01 and PHB2005-0042-PC.

(C)2008 American Mathematical Society Reverts to public domain 28 years from publication 
From now on, $\boldsymbol{a} \otimes \boldsymbol{b}$ denotes the tensorial product matrix of two vectors $\boldsymbol{a}=$ $\left(a_{i}\right)_{i=1}^{2}, \boldsymbol{b}=\left(b_{i}\right)_{i=1}^{2}$, with coefficients $(\boldsymbol{a} \otimes \boldsymbol{b})_{i, j}=a_{i} b_{j}$. We use bold-face letters for vectorial elements.

The first model which we will study was derived and analyzed by Kazhikhov and Smagulov [12]. Assume that $\Psi(\rho)=1$ and that the compressible velocity of the fluid can be decomposed into a potential and an incompressible part (see [3, 4]):

$$
\boldsymbol{v}=\boldsymbol{u}-\lambda \nabla \log \rho \quad \text { with } \quad \nabla \cdot \boldsymbol{u}=0 .
$$

Therefore, system (1) becomes:

$$
\left\{\begin{aligned}
(\rho \boldsymbol{u})_{t}+\nabla \cdot((\rho \boldsymbol{u}-\lambda \nabla \rho) \otimes \boldsymbol{u}-\lambda \boldsymbol{u} \otimes \nabla \rho)-\mu \Delta \boldsymbol{u} & \\
+\lambda^{2} \nabla \cdot\left(\frac{1}{\rho} \nabla \rho \otimes \nabla \rho\right)+\nabla P & =\rho \boldsymbol{f} \text { in } Q, \\
\nabla \cdot \boldsymbol{u}=0 \text { in } Q, \quad \rho_{t}+\nabla \cdot(\rho \boldsymbol{u}-\lambda \nabla \rho) & =0 \quad \text { in } Q,
\end{aligned}\right.
$$

where $P=q-\lambda \rho_{t}+\lambda(2 \mu+\tilde{\lambda}) \Delta \log \rho$. In this paper, we will focus on a simplified version of (3), which is obtained by eliminating the $\lambda^{2}$-term. In fact, using the equalities

$$
(\rho \boldsymbol{u})_{t}+\nabla \cdot((\rho \boldsymbol{u}-\lambda \nabla \rho) \otimes \boldsymbol{u})=\rho \boldsymbol{u}_{t}+((\rho \boldsymbol{u}-\lambda \nabla \rho) \cdot \nabla) \boldsymbol{u}
$$

(thanks to $(3)_{c}$ ), and

$$
\begin{aligned}
-\lambda \nabla \cdot(\boldsymbol{u} \otimes \nabla \rho) & =-\lambda(\boldsymbol{u} \cdot \nabla) \nabla \rho=-\lambda \nabla(\boldsymbol{u} \cdot \nabla \rho)+\lambda \nabla \cdot\left(\rho(\nabla \boldsymbol{u})^{t}\right), \\
\nabla \cdot(\rho \boldsymbol{u}) & =\boldsymbol{u} \cdot \nabla \rho
\end{aligned}
$$

(thanks to $\left.(3)_{b}\right)$, this simplified model is rewritten as follows:

$$
\left\{\begin{array}{l}
\rho \boldsymbol{u}_{t}+((\rho \boldsymbol{u}-\lambda \nabla \rho) \cdot \nabla) \boldsymbol{u}-\nabla \cdot\left(\mu \nabla \boldsymbol{u}-\lambda \rho(\nabla \boldsymbol{u})^{t}\right)+\nabla p=\rho \boldsymbol{f} \quad \text { in } Q \\
\nabla \cdot \boldsymbol{u}=0 \quad \text { in } Q, \quad \rho_{t}+\boldsymbol{u} \cdot \nabla \rho-\lambda \Delta \rho=0 \quad \text { in } Q
\end{array}\right.
$$

where $p=P-\lambda \boldsymbol{u} \cdot \nabla \rho$.

The second model of Kazhikov-Smagulov type which we will consider in this work was analyzed by D. Bresch, E.H. Essoufi and M. Sy ([3]). Such a model can be deduced from (11) imposing (2), $\Psi(\rho)=\rho$ and $\mu=\lambda$, and using again equalities (44)-(6), it is written as:

$$
\left\{\begin{array}{l}
\rho \boldsymbol{u}_{t}+((\rho \boldsymbol{u}-\lambda \nabla \rho) \cdot \nabla) \boldsymbol{u}-\lambda \nabla \cdot\left(\rho \nabla \boldsymbol{u}-\rho(\nabla \boldsymbol{u})^{t}\right)+\nabla p=\rho \boldsymbol{f} \\
\nabla \cdot \boldsymbol{u}=0 \quad \text { in } Q, \quad \rho_{t}+\boldsymbol{u} \cdot \nabla \rho-\lambda \Delta \rho=0 \quad \text { in } Q .
\end{array}\right.
$$

This model is related to a pollution problem [3, 4].

Note that the main differences with respect to the previous system (3) are that the $\lambda^{2}$-terms are all of potential type (included into the modified pressure $p=$ $q-\lambda(\widetilde{\lambda}+\lambda) \Delta \log \rho)$ and the diffusion becomes nonlinear, changing $-\mu \nabla \cdot(\nabla \boldsymbol{u})$ by $-\lambda \nabla \cdot(\rho \nabla \boldsymbol{u})$ in the momentum system.

We complete these models with the following boundary conditions

$$
\boldsymbol{u}_{\left.\right|_{\Sigma}}=0,\left.\quad \frac{\partial \rho}{\partial \boldsymbol{n}}\right|_{\Sigma}=0
$$

(where $\boldsymbol{n}(\boldsymbol{x})$ is the outwards unit normal vector on the boundary $\Gamma$ ) and initial conditions

$$
\rho(\boldsymbol{x}, 0)=\rho_{0}(\boldsymbol{x}), \quad \boldsymbol{u}(\boldsymbol{x}, 0)=\boldsymbol{u}_{0}(\boldsymbol{x}), \quad \boldsymbol{x} \in \Omega .
$$


1.2. Weak solutions. To define the concept of weak solutions, we introduce the following function spaces:

$$
\begin{aligned}
\boldsymbol{H} & =\left\{\boldsymbol{u}: \boldsymbol{u} \in \boldsymbol{L}^{2}(\Omega), \nabla \cdot \boldsymbol{u}=0 \text { in } \Omega, \boldsymbol{u} \cdot \boldsymbol{n}=0 \text { on } \Gamma\right\}, \\
\boldsymbol{V} & =\left\{\boldsymbol{u}: \boldsymbol{u} \in \boldsymbol{H}_{0}^{1}(\Omega), \nabla \cdot \boldsymbol{u}=0 \text { in } \Omega\right\}, \\
L_{0}^{2}(\Omega) & =\left\{p: p \in L^{2}(\Omega), \int_{\Omega} p(\boldsymbol{x}) d \boldsymbol{x}=0\right\}, \\
H_{N}^{2}(\Omega) & =\left\{\rho \in H^{2}(\Omega): \frac{\partial \rho}{\partial \boldsymbol{n}}=0 \text { on } \Gamma, \int_{\Omega} \rho(\boldsymbol{x}) d \boldsymbol{x}=\int_{\Omega} \rho_{0}(\boldsymbol{x}) d \boldsymbol{x}\right\} .
\end{aligned}
$$

In $\boldsymbol{V}$, the $\|\boldsymbol{u}\|_{H^{1}(\Omega)}$ and $\|\nabla \boldsymbol{u}\|_{L^{2}(\Omega)}$ norms are equivalent. $H_{N}^{2}(\Omega)$ is an affine space,

$$
\begin{aligned}
& H_{N}^{2}(\Omega)=\frac{1}{|\Omega|} \int_{\Omega} \rho_{0}(\boldsymbol{x}) d \boldsymbol{x}+H_{N, 0}^{2}(\Omega) \text { where } \\
& \qquad H_{N, 0}^{2}(\Omega)=\left\{\rho \in H^{2}(\Omega): \frac{\partial \rho}{\partial \boldsymbol{n}}=0 \text { on } \partial \Omega, \int_{\Omega} \rho(\boldsymbol{x}) d \boldsymbol{x}=0\right\} .
\end{aligned}
$$

In $H_{N}^{2}(\Omega)$ space, the $\|\nabla \rho\|_{H^{1}(\Omega)}$-norm is equivalent to the $\|\Delta \rho\|_{L^{2}(\Omega)}$-norm.

We denote the norm and the scalar product in $L^{2}(\Omega)$ by $|\cdot|$ and $(\cdot, \cdot)$, respectively, and the norm in $H_{0}^{1}(\Omega)$ of the gradient in $L^{2}(\Omega)$ by $\|\cdot\|$.

Throughout this work, we assume the hypothesis (strictly positive density)

$$
0<m \leq \rho_{0}(\boldsymbol{x}) \leq M \quad \text { in } \Omega .
$$

Definition 1. A pair $(\rho, \boldsymbol{u})$ is called a weak solution of (7), (9)-(10) in $(0, T)$ if:

a) $\boldsymbol{u} \in L^{\infty}(0, T ; \boldsymbol{H}) \cap L^{2}(0, T ; \boldsymbol{V})$, $\rho \in L^{\infty}\left(0, T ; H^{1}(\Omega)\right) \cap L^{2}\left(0, T ; H_{N}^{2}(\Omega)\right)$, $0<m \leq \rho(\boldsymbol{x}, t) \leq M$, a.e. $(\boldsymbol{x}, t) \in Q$.

b) $\forall \phi \in C^{1}([0, T] ; \boldsymbol{V})$ such that $\phi(T)=0$,

$$
\begin{aligned}
& \int_{0}^{T}\left\{-\left(\boldsymbol{u}, \rho \phi_{t}+(\rho \boldsymbol{u}-\lambda \nabla \rho) \cdot \nabla \phi\right)+\left(\mu \nabla \boldsymbol{u}-\lambda \rho(\nabla \boldsymbol{u})^{t}, \nabla \phi\right)\right\} d t \\
& =\int_{0}^{T}(\rho \boldsymbol{f}, \phi) d t+\left(\rho_{0} \boldsymbol{u}_{0}, \phi(0)\right) .
\end{aligned}
$$

c) The equation of mass diffusion (7) $c$ is verified almost everywhere in $Q$.

An analogous definition of weak solution for the second problem (8), (9)-(10) can be made, replacing in Definition 1 the constant viscosity $\mu$ by the dependent-density viscosity $\mu(\rho)=\rho$.

Remark 2. As usual, the pressure $p$ can be obtained by using b) and de Rham's lemma ([17]).

1.3. Known results. The existence and uniqueness of (global in time) weak solution of (7), (9)-(10) was demonstrated in [12, 1].

Theorem 3. Let $\boldsymbol{u}_{0} \in \boldsymbol{H}, \rho_{0} \in H^{1}(\Omega)$ satisfying (11) and $\boldsymbol{f} \in L^{2}\left(0, T ; \boldsymbol{L}^{p}(\Omega)\right)$ with $p>1$. Suppose the constraint on the constants $\lambda, \mu, m$ and $M$ :

$$
\lambda<2 \mu(M-m)^{-1} .
$$

Then, there exists a (unique) weak solution of (7), (9)-(10) in $(0, T)$. 
On the other hand, the existence and uniqueness of a weak solution of (8), (9)(10) was established in [3, 4, without the restrictive hypothesis (12).

Theorem 4. Let $\boldsymbol{u}_{0} \in \boldsymbol{H}$ and $\rho_{0} \in H^{1}(\Omega)$ satisfying (11) and $\boldsymbol{f} \in L^{2}\left(0, T ; \boldsymbol{L}^{p}(\Omega)\right)$ with $p>1$. Then, there exists a (unique) weak solution of (8), (9)-(10) in $(0, T)$.

For the reader's convenience, we will give an outline of both proofs in Appendices A and B, respectively, via a semi-Galerkin method. Here, we introduce a little difference with respect to the proofs given in [12, 1] and [3, 4], which consists in replacing the interpolation inequality $\|\nabla \rho\|_{L^{4}(\Omega)}^{2} \leq C\|\rho\|_{L^{\infty}(\Omega)}|\Delta \rho|$ used to get the $L^{\infty}\left(0, T ; H^{1}(\Omega)\right) \cap L^{2}\left(0, T ; H^{2}(\Omega)\right)$ regularity for the density by the GagliardoNirenberg interpolation inequality in $2 D$ domains $\|\nabla \rho\|_{L^{4}(\Omega)}^{2} \leq C|\nabla \rho||\Delta \rho|$, in order to avoid the use of the maximum principle for the density. Moreover, we think that these proofs will help the reader to understand the statement of our schemes.

Other known results are the following. Salvi ([13]) proved the existence of weak solutions in noncylindrical domains. Secchi ([15]) studied the case $\Omega=\mathbb{R}^{3}$, proving the existence and uniqueness of strong solutions, using a fixed point argument.

With respect to the full model (3), Beirão da Veiga ([2]) and Secchi ([14]) established the local existence of a strong solution by means of linearization and a fixed point argument. In ([14), Secchi proved the existence and uniqueness of a global weak solution in $2 D$ domains imposing smallness on $\lambda / \mu$ and the asymptotic behavior towards a weak solution of the Navier-Stokes problem with variable density. Recently, in [10, by means of an iterative method, the existence of strong solutions (and some error estimates) has been proved.

For the pollutant model (8), Guillén-González and Sy prove the existence of strong solutions of (8) and find some error estimates by means of an iterative method in 11.

From the point of view of numerical analysis, a numerical algorithm is developed in [6, 7, for a compressible version of a Kazhikhov-Smagulov model, without using explicitly the decomposition of the compressible velocity in terms of an incompressible part. The scheme under consideration uses a discrete method of characteristics in time and finite elements in space. The authors get optimal error estimates assuming enough regularity for the continuous solution. It is important to remark that in these works, the analysis of unconditional stable, convergent schemes towards weak solutions is not considered.

1.4. Main results of the paper. The task of this paper is to design fully discrete schemes, unconditionally stable and convergent, by using only $C^{0}$-finite elements for the two problems (77) and (8).

The main question to treat is: Is it possible to approximate the weak solution of mass diffusion problems with only $C^{0}$-finite elements?

The answer is positive for models (7) and (8). Moreover, unconditional stability will be founded.

We will look for schemes using first-order finite difference in time and $C^{0}$-finite elements in space. The key idea is to find an adequate reformulation of the continuous problem, adding "stabilized terms" such that the corresponding Galerkin finite element gives us an unconditionally stable scheme. Namely, in the case of problem (7), (9) and (10), we will arrive at the following variational formulation: 
a.e. $t \in(0, T)$,

$$
\begin{gathered}
\left(\frac{d}{d t} \rho(t), \bar{\rho}\right)+(\boldsymbol{u}(t) \cdot \nabla \rho(t), \bar{\rho})+\lambda(\nabla \rho(t), \nabla \bar{\rho})=0 \quad \forall \bar{\rho} \in H^{1}(\Omega), \\
\left\{\begin{array}{c}
\left([\rho]_{T}(t) \frac{d}{d t} \boldsymbol{u}(t), \overline{\boldsymbol{u}}\right)+\frac{1}{2}\left(\frac{d}{d t}[\rho]_{T}(t) \boldsymbol{u}(t), \overline{\boldsymbol{u}}\right)+a\left([\rho]_{T}(t), \boldsymbol{u}(t), \overline{\boldsymbol{u}}\right) \\
+c(\rho(t) \boldsymbol{u}(t)-\lambda \nabla \rho(t), \boldsymbol{u}(t), \overline{\boldsymbol{u}}) \\
=\left([\rho]_{T}(t) \boldsymbol{f}(t), \overline{\boldsymbol{u}}\right)+(p(t), \nabla \cdot \overline{\boldsymbol{u}}), \quad \forall \overline{\boldsymbol{u}} \in \boldsymbol{H}_{0}^{1}(\Omega), \\
(\nabla \cdot \boldsymbol{u}(t), \bar{p})=0, \quad \forall \bar{p} \in L_{0}^{2}(\Omega),
\end{array}\right.
\end{gathered}
$$

where we have defined

$$
\begin{gathered}
{[\rho]_{T}(\boldsymbol{x}, t)=\left\{\begin{array}{lll}
\rho(\boldsymbol{x}, t) & \text { if } & \rho(\boldsymbol{x}, t) \in[m, M], \\
m & \text { if } & \rho(\boldsymbol{x}, t)<m, \\
M & \text { if } & \rho(\boldsymbol{x}, t)>M,
\end{array}\right.} \\
a(\rho, \boldsymbol{u}, \boldsymbol{v})=\mu(\nabla \boldsymbol{u}, \nabla \boldsymbol{v})+\lambda \int_{\Omega}\left(\frac{M+m}{2}-\rho\right)(\nabla \boldsymbol{u})^{t}: \nabla \boldsymbol{v} d \boldsymbol{x}
\end{gathered}
$$

and

$$
c(\boldsymbol{w}, \boldsymbol{u}, \boldsymbol{v})=\frac{1}{2}[((\boldsymbol{w} \cdot \nabla) \boldsymbol{u}, \boldsymbol{v})-((\boldsymbol{w} \cdot \nabla) \boldsymbol{v}, \boldsymbol{u})],
$$

which verify adequate properties of continuity and coercivity for $a(\cdot, \cdot, \cdot)$ and antisymmetric for $c(\cdot, \cdot, \cdot)$; see (19), (20) and (21) below.

Then, if we choose a partition of $(0, T)$ of parameter $k\left(t_{n}=n k\right)$ and take $\left(W_{h}, \boldsymbol{V}_{h}, M_{h}\right) \subset H^{1} \times \boldsymbol{H}_{0}^{1} \times L_{0}^{2}$ finite-element space for the velocity and the pressure defined below, the following scheme is proposed:

Initialization: Let $\left(\boldsymbol{u}_{h}^{0}, \rho_{h}^{0}\right) \in\left(\boldsymbol{V}_{h}, W_{h}\right)$ be suitable approximations of $\left(\boldsymbol{u}_{0}, \rho_{0}\right)$, as $h \rightarrow 0$.

Time step $(n+1)$ : Given $\left(\boldsymbol{u}_{h}^{n}, p_{h}^{n}, \rho_{h}^{n}\right) \in \boldsymbol{V}_{h} \times M_{h} \times W_{h}$.

(1) Find $\rho_{h}^{n+1} \in W_{h}$ such that for each $\bar{\rho}_{h} \in W_{h}$ :

$$
\left(\frac{\rho_{h}^{n+1}-\rho_{h}^{n}}{k}, \bar{\rho}_{h}\right)+\left(\boldsymbol{u}_{h}^{n} \cdot \nabla \rho_{h}^{n}, \bar{\rho}_{h}\right)+l\left(\nabla \rho_{h}^{n+1}, \nabla \bar{\rho}_{h}\right)=0 .
$$

(2) Find $\left(\boldsymbol{u}_{h}^{n+1}, p_{h}^{n+1}\right) \in \boldsymbol{V}_{h} \times M_{h}$ such that for each $\left(\overline{\boldsymbol{u}}_{h}, \bar{p}_{h}\right) \in \boldsymbol{V}_{h} \times M_{h}$ :

$$
\begin{aligned}
& \left\{\left(\left[\rho_{h}^{n}\right]_{T} \frac{\boldsymbol{u}_{h}^{n+1}-\boldsymbol{u}_{h}^{n}}{k}, \overline{\boldsymbol{u}}_{h}\right)+\frac{1}{2}\left(\frac{\left[\rho_{h}^{n+1}\right]_{T}-\left[\rho_{h}^{n}\right]_{T}}{k}, \boldsymbol{u}_{h}^{n+1} \cdot \overline{\boldsymbol{u}}_{h}\right)+a\left(\left[\rho_{h}^{n+1}\right]_{T}, \boldsymbol{u}_{h}^{n+1}, \overline{\boldsymbol{u}}_{h}\right)\right. \\
& \left(+c\left(\rho_{h}^{n+1} \boldsymbol{u}_{h}^{n}-\lambda \nabla \rho_{h}^{n+1}, \boldsymbol{u}_{h}^{n+1}, \overline{\boldsymbol{u}}_{h}\right)=\left(\left[\rho_{h}^{n+1}\right]_{T} \boldsymbol{f}^{n+1}, \overline{\boldsymbol{u}}_{h}\right)+\left(p_{h}^{n+1}, \nabla \cdot \overline{\boldsymbol{u}}_{h}\right),\right. \\
& \left(\nabla \cdot \boldsymbol{u}_{h}^{n+1}, \bar{p}_{h}\right)=0 \text {. }
\end{aligned}
$$

Defining in $[0, T]$ the functions $\boldsymbol{u}_{k, h}, \rho_{h, k}$ as constant by subintervals such that $\boldsymbol{u}_{k, h}(t)=\boldsymbol{u}_{h}^{n}$ and $\rho_{h, k}(t)=\rho_{h}^{n}$ on $\left(t_{n-1}, t_{n}\right]$, respectively, we arrive at the following main result:

Theorem 5. Let $\boldsymbol{u}_{0} \in \boldsymbol{V}, \rho_{0} \in H_{N}^{2}(\Omega)$ satisfying (11) and $\boldsymbol{f} \in L^{2}\left(0, T ; \boldsymbol{L}^{p}(\Omega)\right)$ with $p>1$. Suppose the constraint on the constants $\lambda, \mu, m$ and $M: \lambda<$ $2 \mu(M-m)^{-1}$. Then, the whole sequence $\left(\boldsymbol{u}_{k, h}, \rho_{h, k}\right)$ converges towards the (unique) weak solution $(\boldsymbol{u}, \rho)$ of problem (7), (9) and (10) (see Definition 1), strongly in 
$L^{2}\left(0, T ; \boldsymbol{L}^{2}(\Omega) \times H^{1}(\Omega)\right)$, weak-star in $L^{\infty}\left(0, T ; \boldsymbol{L}^{2}(\Omega) \times H^{1}(\Omega)\right)$ and weakly in $L^{2}\left(0, T ; \boldsymbol{H}_{0}^{1}(\Omega)\right) \times L^{4}\left(0, T ; W^{1,4}(\Omega)\right)$.

Remark 6 . The term $c\left(\rho_{h}^{n+1} \boldsymbol{u}_{h}^{n}-\lambda \nabla \rho_{h}^{n+1}, \boldsymbol{u}_{h}^{n+1}, \overline{\boldsymbol{u}}_{h}\right)$ of the velocity-pressure scheme can be changed by $c\left(\rho_{h}^{n} \boldsymbol{u}_{h}^{n}-\lambda \nabla \rho_{h}^{n+1}, \boldsymbol{u}_{h}^{n+1}, \overline{\boldsymbol{u}}_{h}\right)$, keeping all results of this paper.

After a justification of the choice of the scheme made in Section 2, we give the proof of the previous convergence theorem from Section 3 to Section 6. An analogous result for the second problem (8), (9)-(10) will also be obtained in Section 7. Moreover, a generalization of this second model will be presented in Section 8, obtaining an unconditional stable scheme, but its convergence remains as an open problem.

\section{DESIGN OF THE NUMERICAL SCHEME}

This section is devoted to designing an unconditionally stable, convergent scheme, using the backward Euler scheme in time (considering for simplicity a uniform partition of $[0, T]$ with time step $\left.k=T / N:\left(t_{n}=n k\right)_{n=0}^{n=N}\right)$, and finite elements in space.

In order to get an easy implementation, we are going to define a linear scheme with decoupled problems with respect to $(\boldsymbol{u}, p)$ and $\rho$ in each time step. Concerning the space discretization, we only choose at most $H^{1}$-conformed finite element spaces for the density, velocity and pressure, which we denote by $\left(W_{h}, \boldsymbol{V}_{h}, M_{h}\right) \subset H^{1} \times$ $\boldsymbol{H}_{0}^{1} \times L_{0}^{2}$ with the density space $W_{h}$ generated by $\mathbb{P}_{1}$ continuous finite elements and velocity-pressure spaces $\left(\boldsymbol{V}_{h}, M_{h}\right)$ satisfying the stability Babuska-Bezzi condition ([8]).

To start these requirements, a first attempt would be the following scheme.

Let $\rho_{h}^{n} \in W_{h}$ and $\boldsymbol{u}_{h}^{n} \in \boldsymbol{V}_{h}$ be given.

(1) Find $\rho_{h}^{n+1} \in W_{h}$ such that for each $\bar{\rho}_{h} \in W_{h}$ :

$$
\left(\frac{\rho_{h}^{n+1}-\rho_{h}^{n}}{k}, \bar{\rho}_{h}\right)+\left(\boldsymbol{u}_{h}^{n} \cdot \nabla \rho_{h}^{n}, \bar{\rho}_{h}\right)+\lambda\left(\nabla \rho_{h}^{n+1}, \nabla \bar{\rho}_{h}\right)=0
$$

(2) Find $\left(\boldsymbol{u}_{h}^{n+1}, p_{h}^{n+1}\right) \in \boldsymbol{V}_{h} \times M_{h}$ such that for each $\left(\overline{\boldsymbol{u}}_{h}, \bar{p}_{h}\right) \in \boldsymbol{V}_{h} \times M_{h}$ :

$$
\begin{aligned}
& \left\{\begin{array}{l}
\left(\rho_{h}^{n} \frac{\boldsymbol{u}_{h}^{n+1}-\boldsymbol{u}_{h}^{n}}{k}, \overline{\boldsymbol{u}}_{h}\right)+\left(\left(\left(\rho_{h}^{n+1} \boldsymbol{u}_{h}^{n}-\lambda \nabla \rho_{h}^{n+1}\right) \cdot \nabla\right) \boldsymbol{u}_{h}^{n+1}, \overline{\boldsymbol{u}}_{h}\right)-\left(p_{h}^{n+1}, \nabla \cdot \overline{\boldsymbol{u}}_{h}\right) \\
+\left(\mu \nabla \boldsymbol{u}_{h}^{n+1}-\lambda \rho_{h}^{n+1}\left(\nabla \boldsymbol{u}_{h}^{n+1}\right)^{t}, \nabla \overline{\boldsymbol{u}}_{h}\right)=\left(\rho_{h}^{n+1} \boldsymbol{f}^{n+1}, \overline{\boldsymbol{u}}_{h}\right)
\end{array}\right. \\
& \left(\begin{array}{c}
\left(\nabla \cdot \boldsymbol{u}_{h}^{n+1}, \bar{p}_{h}\right)=0
\end{array}\right.
\end{aligned}
$$

where $\boldsymbol{f}^{n+1}=\frac{1}{k} \int_{t_{n}}^{t_{n+1}} \boldsymbol{f}(t) d t$. 
Remark 7. The approximation of $\left(\rho \boldsymbol{u}_{t}\right)\left(t_{n+1}\right)$ by $\rho_{h}^{n} \frac{\boldsymbol{u}_{h}^{n+1}-\boldsymbol{u}_{h}^{n}}{k}$ is justified for the equality:

$$
\begin{aligned}
& \left(\rho_{h}^{n} \frac{\boldsymbol{u}_{h}^{n+1}-\boldsymbol{u}_{h}^{n}}{k}, \boldsymbol{u}_{h}^{n+1}\right)+\frac{1}{2}\left(\frac{\rho_{h}^{n+1}-\rho_{h}^{n}}{k}, \boldsymbol{u}_{h}^{n+1} \cdot \boldsymbol{u}_{h}^{n+1}\right) \\
& =\frac{1}{2}\left(\int_{\Omega} \frac{\rho_{h}^{n+1}\left|\boldsymbol{u}_{h}^{n+1}\right|^{2}-\rho_{h}^{n}\left|\boldsymbol{u}_{h}^{n}\right|^{2}}{k}+\int_{\Omega} \frac{\rho_{h}^{n}\left|\boldsymbol{u}_{h}^{n+1}-\boldsymbol{u}_{h}^{n}\right|^{2}}{k}\right),
\end{aligned}
$$

which is a discrete version of the continuous relation:

$$
\left(\rho \frac{d}{d t} \boldsymbol{u}, \boldsymbol{u}\right)+\frac{1}{2}\left(\frac{d}{d t} \rho, \boldsymbol{u} \cdot \boldsymbol{u}\right)=\frac{1}{2} \frac{d}{d t} \int_{\Omega} \rho|\boldsymbol{u}|^{2} .
$$

Throughout this work we assume the following hypotheses:

(H1) Either

$$
\boldsymbol{u}_{0} \in \boldsymbol{H} \text { and } \rho_{0} \in H_{N}^{1}(\Omega) \text { with } k / h^{2} \leq C
$$

or

$$
\boldsymbol{u}_{0} \in \boldsymbol{V} \text { and } \rho_{0} \in H_{N}^{2}(\Omega)
$$

(H2) The boundary of $\Omega$ is a polygon such that the continuous dependency in the $H^{2}$-norm of the Poisson-Neumann problem holds (see (29)). This is true, for instance, if $\Omega$ is convex $([])$.

(H3) The triangulation of $\Omega$ and the discrete spaces. Let $\left\{\mathcal{T}_{h}\right\}_{h>0}$ be a regular, quasi-uniform family of triangulations of $\Omega$, with $h=\max _{K \in \mathcal{T}_{h}} h_{K}\left(h_{K}=\right.$ diameter of $K$ ), and

$$
W_{h}=\left\{x_{h} \in C^{0}(\bar{\Omega}):\left.x_{h}\right|_{K} \in \mathbb{P}_{1}(K), \forall K \in \mathcal{T}_{h}\right\} .
$$

In particular, this discrete space verifies the following properties (5]) which we are going to use in this paper:

- the inverse inequalities:

$$
\begin{aligned}
\left\|\nabla \bar{\rho}_{h}\right\|_{L^{4}(\Omega)} & \leq C h^{-1 / 2}\left|\nabla \bar{\rho}_{h}\right|, \quad \forall \bar{\rho}_{h} \in W_{h}, \\
\left\|\bar{\rho}_{h}\right\|_{H^{1}(\Omega)} & \leq C h^{-1}\left|\bar{\rho}_{h}\right|, \quad \forall \bar{\rho}_{h} \in W_{h},
\end{aligned}
$$

- and the interpolation errors:

$$
\begin{aligned}
& \left|\bar{\rho}-I_{h} \bar{\rho}\right| \leq C h\|\bar{\rho}\|_{H^{1}(\Omega)}, \\
& \left\|\bar{\rho}-I_{h} \bar{\rho}\right\|_{H^{1}(\Omega)}+h^{1 / 2}\left\|\bar{\rho}-I_{h} \bar{\rho}\right\|_{W^{1,4}(\Omega)} \leq C h\|\bar{\rho}\|_{H^{2}(\Omega)}, \quad \forall \bar{\rho} \in H^{2}(\Omega),
\end{aligned}
$$

where $I_{h}$ is the interpolation operator from $H^{2}(\Omega)$ into $W_{h}$.

On the other hand, we choose $\left(V_{h}, M_{h}\right)$ verifying the interpolation errors:

$$
\begin{aligned}
& \left|\overline{\boldsymbol{u}}-J_{h} \overline{\boldsymbol{u}}\right|+h\left\|\overline{\boldsymbol{u}}-J_{h} \overline{\boldsymbol{u}}\right\|_{H^{1}(\Omega)} \leq C h^{2}\|\overline{\boldsymbol{u}}\|_{H^{2}(\Omega)}, \quad \forall \overline{\boldsymbol{u}} \in \boldsymbol{H}^{2}(\Omega) \cap \boldsymbol{H}_{0}^{1}(\Omega), \\
& \left|\bar{p}-K_{h} \bar{p}\right| \leq C h\|\bar{p}\|_{H^{1}(\Omega)}, \quad \forall \bar{p} \in H^{1}(\Omega) \cap L_{0}^{2}(\Omega),
\end{aligned}
$$

where $J_{h}$ and $K_{h}$ are interpolation operators from $\boldsymbol{H}^{2}(\Omega) \cap \boldsymbol{H}_{0}^{1}(\Omega)$ into $\boldsymbol{V}_{h}$ and from $H^{1}(\Omega) \cap L_{0}^{2}(\Omega)$ into $M_{h}$, respectively.

(H4) Inf-sup condition. There is a constant $\beta>0$ (independent of $h$ ) such that $\forall \bar{p}_{h} \in M_{h}$,

$$
\left\|\bar{p}_{h}\right\|_{L_{0}^{2}(\Omega)} \leq \beta \sup _{\overline{\boldsymbol{u}}_{h} \in \boldsymbol{V}_{h} \backslash\{0\}} \frac{\left(\bar{p}_{h}, \nabla \cdot \overline{\boldsymbol{u}}_{h}\right)}{\left\|\overline{\boldsymbol{u}}_{h}\right\|_{H^{1}(\Omega)}} .
$$


For instance, a manner of defining the discrete spaces $\left(\boldsymbol{V}_{h}, M_{h}\right)$ verifying (H3) and $(\mathrm{H} 4)$ is:

$$
M_{h}=W_{h} \cap L_{0}^{2}(\Omega)
$$

and to select $\boldsymbol{V}_{h}$ there are several possibilities ([8]). For instance:

(1) (Taylor-Hood)

$$
\boldsymbol{V}_{h}=\left\{v_{h} \in C^{0}(\bar{\Omega}):\left.v_{h}\right|_{K} \in \mathbb{P}_{2}(K), \forall K \in \mathcal{T}_{h}\right\}^{2} \cap \boldsymbol{H}_{0}^{1}(\Omega) .
$$

(2) (Mini-element) Define $\mathcal{P}(K)=\left[\mathbb{P}_{1}(K)\right]^{2} \oplus \alpha_{K} \lambda_{1} \lambda_{2} \lambda_{3}$ with $\alpha_{K} \in \mathbb{R}^{2}$ and $\lambda_{i} \in \mathbb{P}_{1}$ such that $\lambda_{i}\left(a_{j}\right)=\delta_{i j}, a_{j}$ being the vertices of the triangle $K$. Then, we consider

$$
\boldsymbol{V}_{h}=\left\{v_{h} \in C^{0}(\bar{\Omega}):\left.v_{h}\right|_{K} \in \mathcal{P}(K), \forall K \in \mathcal{T}_{h}\right\}^{2} \cap \boldsymbol{H}_{0}^{1}(\Omega) .
$$

To obtain estimates of scheme (13)-(15), the idea is to follow the proof of the existence Theorem 3 (see Appendix $\mathrm{A}$ ), but we find the following main difficulties:

(1) We cannot assure the maximum principle for the discrete density $\rho_{h}^{n}$.

(2) The density equation doesn't hold pointwise (as used in the proof of Theorem 3) see Appendix (A), or more concretely, we cannot take $\bar{\rho}_{h}=\frac{1}{2} \boldsymbol{u}_{h}^{n+1}$. $\boldsymbol{u}_{h}^{n+1}$ in (13), because in general $\boldsymbol{u}_{h}^{n+1} \cdot \boldsymbol{u}_{h}^{n+1} \notin W_{h}$.

(3) The incompressibility condition doesn't hold pointwise; therefore

$$
\int_{\Omega}\left(\nabla \boldsymbol{u}_{h}^{n+1}\right)^{t}: \nabla \boldsymbol{u}_{h}^{n+1} \neq 0
$$

in general.

(4) We are not going to get strong $H^{2}$ estimates for the discrete density $\rho_{h}^{n}$, since we are approximating in $H^{1}$ (or at the most in $W^{1, \infty}$ ), but not in $H^{2}$.

To treat difficulty (1), i.e. the absence of the maximum principle, we define the following truncating (by nodes) operator: Given $w_{h} \in W_{h}$, one defines $\left[w_{h}\right]_{T} \in W_{h}$ such that:

$$
\left[w_{h}\right]_{T}\left(\boldsymbol{x}_{i}\right)=\left\{\begin{array}{lll}
w_{h}\left(\boldsymbol{x}_{i}\right) & \text { if } & w_{h}\left(\boldsymbol{x}_{i}\right) \in[m, M], \\
m & \text { if } & w_{h}\left(\boldsymbol{x}_{i}\right)<m, \\
M & \text { if } & w_{h}\left(\boldsymbol{x}_{i}\right)>M,
\end{array}\right.
$$

where $\boldsymbol{x}_{i}$ are the nodes of the mesh $\mathcal{T}_{h}$.

To treat difficulty (2), we add to the discrete momentum system (14) the following terms:

$$
\frac{1}{2}\left(\frac{\left[\rho_{h}^{n+1}\right]_{T}-\left[\rho_{h}^{n}\right]_{T}}{k}, \boldsymbol{u}_{h}^{n+1} \cdot \overline{\boldsymbol{u}}_{h}\right)-\frac{1}{2}\left(\rho_{h}^{n+1} \boldsymbol{u}_{h}^{n}-\lambda \nabla \rho_{h}^{n+1}, \nabla\left(\boldsymbol{u}_{h}^{n+1} \cdot \overline{\boldsymbol{u}}_{h}\right)\right),
$$

where we have only truncated the discrete densities in the first term. Then, we change (14) by

$$
\left\{\begin{aligned}
& \left(\left[\rho_{h}^{n}\right]_{T} \frac{\boldsymbol{u}_{h}^{n+1}-\boldsymbol{u}_{h}^{n}}{k}, \overline{\boldsymbol{u}}_{h}\right)+\frac{1}{2}\left(\frac{\left[\rho_{h}^{n+1}\right]_{T}-\left[\rho_{h}^{n}\right]_{T}}{k}, \boldsymbol{u}_{h}^{n+1} \cdot \overline{\boldsymbol{u}}_{h}\right) \\
+ & \left(\left(\left(\rho_{h}^{n+1} \boldsymbol{u}_{h}^{n}-\lambda \nabla \rho_{h}^{n+1}\right) \cdot \nabla\right) \boldsymbol{u}_{h}^{n+1}, \overline{\boldsymbol{u}}_{h}\right)-\frac{1}{2}\left(\rho_{h}^{n+1} \boldsymbol{u}_{h}^{n}-\lambda \nabla \rho_{h}^{n+1}, \nabla\left(\boldsymbol{u}_{h}^{n+1} \cdot \overline{\boldsymbol{u}}_{h}\right)\right) \\
+ & \left(\mu \nabla \boldsymbol{u}_{h}^{n+1}-\lambda\left[\rho_{h}^{n+1}\right]_{T}\left(\nabla \boldsymbol{u}_{h}^{n+1}\right)^{t}, \nabla \overline{\boldsymbol{u}}_{h}\right)=\left(\left[\rho_{h}^{n+1}\right]_{T} \boldsymbol{f}^{n+1}, \overline{\boldsymbol{u}}_{h}\right)+\left(p_{h}^{n+1}, \nabla \cdot \overline{\boldsymbol{u}}_{h}\right),
\end{aligned}\right.
$$

where we have also truncated the discrete density in the terms

$$
\int_{\Omega} \rho_{h}^{n+1}\left(\nabla \boldsymbol{u}_{h}^{n+1}\right)^{t}: \nabla \overline{\boldsymbol{u}}_{h} d \boldsymbol{x} \quad \text { and } \quad\left(\rho_{h}^{n+1} \boldsymbol{f}^{n+1}, \overline{\boldsymbol{u}}_{h}\right) .
$$


This last truncation is considered to reduce the hypothesis on the external force $f$ acting on the system.

With respect to difficulty (3), in order to "control" the term

$$
-\lambda \int_{\Omega}\left[\rho_{h}^{n+1}\right]_{T}\left(\nabla \boldsymbol{u}_{h}^{n+1}\right)^{t}:\left(\nabla \overline{\boldsymbol{u}}_{h}\right) d \boldsymbol{x}
$$

we add to (16) the term:

$$
\lambda \int_{\Omega} \frac{M+m}{2}\left(\nabla \boldsymbol{u}_{h}^{n+1}\right)^{t}:\left(\nabla \overline{\boldsymbol{u}}_{h}\right) d \boldsymbol{x} .
$$

Then, taking $\overline{\boldsymbol{u}}_{h}=\boldsymbol{u}_{h}^{n+1}$ in (16), we obtain the following estimate (equivalent to (85)):

$$
\left|\lambda \int_{\Omega}\left(\left[\rho_{h}^{n+1}\right]_{T}-\frac{M+m}{2}\right)\left(\nabla \boldsymbol{u}_{h}^{n+1}\right)^{t}:\left(\nabla \boldsymbol{u}_{h}^{n+1}\right) d \boldsymbol{x}\right| \leq \lambda \frac{M-m}{2}\left\|\boldsymbol{u}_{h}^{n+1}\right\|^{2} .
$$

On the other hand, applying Leibnitz' rule in the term

$$
-\frac{1}{2}\left(\rho_{h}^{n+1} \boldsymbol{u}_{h}^{n}-\lambda \nabla \rho_{h}^{n+1}, \nabla\left(\boldsymbol{u}_{h}^{n+1} \cdot \overline{\boldsymbol{u}}_{h}\right)\right),
$$

we rewrite (16) as:

(18)

$$
\left\{\begin{array}{l}
\left(\left[\rho_{h}^{n}\right]_{T} \frac{\boldsymbol{u}_{h}^{n+1}-\boldsymbol{u}_{h}^{n}}{k}, \overline{\boldsymbol{u}}_{h}\right)+\frac{1}{2}\left(\frac{\left[\rho_{h}^{n+1}\right]_{T}-\left[\rho_{h}^{n}\right]_{T}}{k}, \boldsymbol{u}_{h}^{n+1} \cdot \overline{\boldsymbol{u}}_{h}\right)+a\left(\left[\rho_{h}^{n+1}\right]_{T}, \boldsymbol{u}_{h}^{n+1}, \overline{\boldsymbol{u}}_{h}\right) \\
+c\left(\rho_{h}^{n+1} \boldsymbol{u}_{h}^{n}-\lambda \nabla \rho_{h}^{n+1}, \boldsymbol{u}_{h}^{n+1}, \overline{\boldsymbol{u}}_{h}\right)=\left(\left[\rho_{h}^{n+1}\right]_{T} \boldsymbol{f}^{n+1}, \overline{\boldsymbol{u}}_{h}\right)+\left(p_{h}^{n+1}, \nabla \cdot \overline{\boldsymbol{u}}_{h}\right),
\end{array}\right.
$$

where we have defined

$$
a(\rho, \boldsymbol{u}, \boldsymbol{v})=\mu(\nabla \boldsymbol{u}, \nabla \boldsymbol{v})+\lambda \int_{\Omega}\left(\frac{M+m}{2}-\rho\right)(\nabla \boldsymbol{u})^{t}: \nabla \boldsymbol{v} d \boldsymbol{x}
$$

and

$$
c(\boldsymbol{w}, \boldsymbol{u}, \boldsymbol{v})=\frac{1}{2}[((\boldsymbol{w} \cdot \nabla) \boldsymbol{u}, \boldsymbol{v})-((\boldsymbol{w} \cdot \nabla) \boldsymbol{v}, \boldsymbol{u})],
$$

which verify the properties:

$$
\begin{gathered}
a\left([\rho]_{T}, \boldsymbol{u}, \boldsymbol{u}\right) \geq \frac{\mu_{1}}{2}\|\boldsymbol{u}\|^{2} \quad \text { where } \frac{\mu_{1}}{2}=\mu-\lambda \frac{M-m}{2}(>0), \quad(\text { using (12) }), \\
a\left([\rho]_{T}, \boldsymbol{u}, \boldsymbol{v}\right) \leq C\|\boldsymbol{u}\|\|\boldsymbol{v}\| \quad\left(\text { using }\left\|[\rho]_{T}\right\|_{L^{\infty}(\Omega)} \leq M\right), \\
c(\boldsymbol{w}, \boldsymbol{u}, \boldsymbol{u})=0, \\
c(\boldsymbol{w}, \boldsymbol{u}, \boldsymbol{v}) \leq C\|\boldsymbol{w}\|_{L^{3}(\Omega)}\|\boldsymbol{u}\|\|\boldsymbol{v}\| .
\end{gathered}
$$

Finally, we will see that difficulty (4) can be circumvented.

In conclusion, we define the following numerical scheme:

Initialization: Let $\left(\boldsymbol{u}_{h}^{0}, \rho_{h}^{0}\right) \in\left(\boldsymbol{V}_{h}, W_{h}\right)$ be suitable approximations of $\left(\boldsymbol{u}_{0}, \rho_{0}\right)$, as $h \rightarrow 0$.

Time step $(n+1)$ : Given $\left(\boldsymbol{u}_{h}^{n}, p_{h}^{n}, \rho_{h}^{n}\right) \in \boldsymbol{V}_{h} \times M_{h} \times W_{h}$.

(1) Find $\rho_{h}^{n+1} \in W_{h}$ such that for each $\bar{\rho}_{h} \in W_{h}$ :

$$
\left(\frac{\rho_{h}^{n+1}-\rho_{h}^{n}}{k}, \bar{\rho}_{h}\right)+\left(\boldsymbol{u}_{h}^{n} \cdot \nabla \rho_{h}^{n}, \bar{\rho}_{h}\right)+\lambda\left(\nabla \rho_{h}^{n+1}, \nabla \bar{\rho}_{h}\right)=0
$$


(2) Find $\left(\boldsymbol{u}_{h}^{n+1}, p_{h}^{n+1}\right) \in \boldsymbol{V}_{h} \times M_{h}$ such that for each $\left(\overline{\boldsymbol{u}}_{h}, \bar{p}_{h}\right) \in \boldsymbol{V}_{h} \times M_{h}$ :

$$
\begin{gathered}
\left\{\begin{array}{c}
\left(\left[\rho_{h}^{n}\right]_{T} \frac{\boldsymbol{u}_{h}^{n+1}-\boldsymbol{u}_{h}^{n}}{k}, \overline{\boldsymbol{u}}_{h}\right)+\frac{1}{2}\left(\frac{\left[\rho_{h}^{n+1}\right]_{T}-\left[\rho_{h}^{n}\right]_{T}}{k}, \boldsymbol{u}_{h}^{n+1} \cdot \overline{\boldsymbol{u}}_{h}\right)+a\left(\left[\rho_{h}^{n+1}\right]_{T}, \boldsymbol{u}_{h}^{n+1}, \overline{\boldsymbol{u}}_{h}\right) \\
+c\left(\rho_{h}^{n+1} \boldsymbol{u}_{h}^{n}-\lambda \nabla \rho_{h}^{n+1}, \boldsymbol{u}_{h}^{n+1}, \overline{\boldsymbol{u}}_{h}\right)=\left(\left[\rho_{h}^{n+1}\right]_{T} \boldsymbol{f}^{n+1}, \overline{\boldsymbol{u}}_{h}\right)+\left(p_{h}^{n+1}, \nabla \cdot \overline{\boldsymbol{u}}_{h}\right),
\end{array}\right. \\
\left(\nabla \cdot \boldsymbol{u}_{h}^{n+1}, \bar{p}_{h}\right)=0 .
\end{gathered}
$$

From the computational point of view, we propose a scheme where in each time step we have to solve two (decoupled) linear systems: the diffusion problem (22) for $\rho_{h}^{n+1}$ and the problem of Stokes type (23)-(24) for $\left(\boldsymbol{u}_{h}^{n+1}, p_{h}^{n+1}\right)$.

To conclude this section, we shall see that the linear systems (22) and (23)-(24) are well-posed; that is, the existence and uniqueness of a solution hold. Indeed, as they can be written as algebraic linear systems, it suffices to verify the uniqueness of a solution for each problem, which will be deduced in particular from the a priori scheme estimates of the next section.

\section{A priori SChEMe Estimates (UnConditional stability)}

In order to get stability estimates in strong norms for the density, we will need a discrete version of the $2 D$ inequality interpolation $\|\nabla \rho\|_{L^{4}(\Omega)}^{2} \leq C|\nabla \rho \| \Delta \rho|$. For this, we first introduce the discrete Laplacian using the following auxiliary problem:

Given $\left(\boldsymbol{u}_{h}^{n}, \rho_{h}^{n}\right) \in \boldsymbol{V}_{h} \times W_{h}$, find $\left(\rho_{h}^{n+1}, \omega_{h}^{n+1}\right) \in W_{h} \times W_{h}$ such that:

$$
\left\{\begin{array}{l}
\left(\frac{\rho_{h}^{n+1}-\rho_{h}^{n}}{k}, \bar{\omega}_{h}\right)+\left(\boldsymbol{u}_{h}^{n} \cdot \nabla \rho_{h}^{n}, \bar{\omega}_{h}\right)+\lambda\left(\omega_{h}^{n+1}, \bar{\omega}_{h}\right)=0, \\
\left(\nabla \rho_{h}^{n+1}, \nabla \bar{\rho}_{h}\right)=\left(\omega_{h}^{n+1}, \bar{\rho}_{h}\right),
\end{array}\right.
$$

for each $\left(\bar{\omega}_{h}, \bar{\rho}_{h}\right) \in W_{h} \times W_{h}$. It is easy to prove that (25) has a unique solution.

Lemma 8. Problems (25) and (22) are equivalent.

Proof. Suppose that $\left(\rho_{h}^{n+1}, \omega_{h}^{n+1}\right)$ is the solution of (25). Then, replacing (25) $b$ into (25) $a$ for $\bar{\rho}_{h}=\bar{\omega}_{h}$, this gives that $\rho_{h}^{n+1}$ is solution of (22).

On the other hand, suppose that $\rho_{h}^{n+1}$ is the solution of (22). We shall define $\omega_{h}^{n+1} \in W_{h}$ as the solution of:

$$
\lambda\left(\omega_{h}^{n+1}, \bar{\omega}_{h}\right)=-\left(\frac{\rho_{h}^{n+1}-\rho_{h}^{n}}{k}, \bar{\omega}_{h}\right)-\left(\boldsymbol{u}_{h}^{n} \cdot \nabla \rho_{h}^{n}, \bar{\omega}_{h}\right), \quad \forall \bar{\omega}_{h} \in W_{h} .
$$

Comparing (22) and (26) for $\bar{\rho}_{h}=\bar{\omega}_{h}$, we arrive at

$$
\left(\nabla \rho_{h}^{n+1}, \nabla \bar{\rho}_{h}\right)=\left(\omega_{h}^{n+1}, \bar{\rho}_{h}\right), \quad \forall \bar{\rho}_{h} \in W_{h} .
$$

Consequently $\left(\rho_{h}^{n+1}, \omega_{h}^{n+1}\right)$ is the solution of (25) .

Remark 9. Taking $\bar{\rho}_{h}=1$ as a test function in $(25)_{b}$, one has that $\int_{\Omega} \omega_{h}^{n+1}=0$.

Lemma 10. Let $\omega_{h} \in W_{h} \cap L_{0}^{2}(\Omega)$ and $\rho_{h} \in W_{h}$ such that:

$$
\left(\nabla \rho_{h}, \nabla \bar{\rho}_{h}\right)=\left(\omega_{h}, \bar{\rho}_{h}\right), \quad \forall \bar{\rho}_{h} \in W_{h} .
$$


Then, there exists $C>0$ (independent of $h$ ) such that:

$$
\left\|\nabla \rho_{h}\right\|_{L^{4}(\Omega)}^{2} \leq C\left|\nabla \rho_{h}\right|\left|\omega_{h}\right| .
$$

Proof. We define $\rho(h) \in H^{2}(\Omega)$ as the solution of the following continuous problem:

$$
-\Delta \rho(h)=\omega_{h} \text { in } \Omega,\left.\quad \frac{\partial \rho(h)}{\partial \boldsymbol{n}}\right|_{\partial \Omega}=0, \quad \int_{\Omega} \rho(h) d \boldsymbol{x}=0 .
$$

Since the compatibility condition $\int_{\Omega} \omega_{h}=0$ holds, problem (29) is well-posed and verifies the continuous dependency property

$$
\|\rho(h)\|_{H^{2}(\Omega)} \leq C\left|\omega_{h}\right| .
$$

Now, we decompose

$$
\begin{aligned}
\left\|\nabla \rho_{h}\right\|_{L^{4}(\Omega)} \leq \| & \nabla \rho_{h}-\nabla I_{h}(\rho(h)) \|_{L^{4}(\Omega)} \\
& +\left\|\nabla I_{h}(\rho(h))-\nabla \rho(h)\right\|_{L^{4}(\Omega)}+\|\nabla \rho(h)\|_{L^{4}(\Omega)}
\end{aligned}
$$

where $I_{h}$ is the interpolation operator from $H^{1}(\Omega)$ into $W_{h}$. By approximation properties of this interpolation operator $([5])$,

$$
\left\|\nabla I_{h}(\rho(h))-\nabla \rho(h)\right\|_{L^{4}(\Omega)} \leq C h^{1 / 2}\|\rho(h)\|_{H^{2}(\Omega)} \leq C h^{1 / 2}\left|\omega_{h}\right| .
$$

Multiplying (29) by $\bar{\rho}_{h} \in W_{h}$ and integrating by parts,

$$
\left(\nabla \rho(h), \nabla \bar{\rho}_{h}\right)=\left(\omega_{h}, \bar{\rho}_{h}\right) .
$$

Comparing (32) and (25) $b$, one gets

$$
\left(\nabla \rho_{h}-\nabla \rho(h), \nabla \bar{\rho}_{h}\right)=0, \quad \forall \bar{\rho}_{h} \in W_{h} .
$$

Adding and subtracting $\nabla I_{h}(\rho(h))$ and considering $\bar{\rho}_{h}=\rho_{h}-I_{h}(\rho(h)) \in W_{h}$, we obtain

$$
\begin{aligned}
\left|\nabla \rho_{h}-\nabla I_{h}(\rho(h))\right|^{2} & =-\left(\nabla I_{h}(\rho(h))-\nabla \rho(h), \nabla \rho_{h}-\nabla I_{h}(\rho(h))\right) \\
& \leq\left|\nabla I_{h}(\rho(h))-\nabla \rho(h)\right|\left|\nabla \rho_{h}-\nabla I_{h}(\rho(h))\right|,
\end{aligned}
$$

whence

$$
\left|\nabla \rho_{h}-\nabla I_{h}(\rho(h))\right| \leq\left|\nabla I_{h}(\rho(h))-\nabla \rho(h)\right| \leq C h\|\rho(h)\|_{H^{2}(\Omega)} \leq C h\left|\omega_{h}\right| .
$$

Therefore, using the inverse inequality $([\underline{[}])$

$$
\left\|\nabla \rho_{h}-\nabla I_{h}(\rho(h))\right\|_{L^{4}(\Omega)} \leq C h^{-1 / 2}\left|\nabla \rho_{h}-\nabla I_{h}(\rho(h))\right|
$$

and (33), we arrive at

$$
\left\|\nabla \rho_{h}-\nabla I_{h}(\rho(h))\right\|_{L^{4}(\Omega)} \leq C h^{1 / 2}\left|\omega_{h}\right| .
$$

Using Gagliardo-Nirenberg's inequality in $2 D$ domains,

$$
\|\nabla \rho(h)\|_{L^{4}(\Omega)} \leq C|\nabla \rho(h)|^{1 / 2}\|\rho(h)\|_{H^{2}(\Omega)}^{1 / 2} \leq C|\nabla \rho(h)|^{1 / 2}\left|\omega_{h}\right|^{1 / 2} .
$$

Applying (31), (34) and (35) in (30), one obtains

$$
\left\|\nabla \rho_{h}\right\|_{L^{4}(\Omega)} \leq C h^{1 / 2}\left|\omega_{h}\right|+C|\nabla \rho(h)|^{1 / 2}\left|\omega_{h}\right|^{1 / 2} .
$$

From (33) and from the interpolation error $\left|\nabla I_{h}(\rho(h))-\nabla \rho(h)\right| \leq C h\|\rho(h)\|_{H^{2}(\Omega)}$, one has

$$
\left|\nabla \rho_{h}-\nabla \rho(h)\right| \leq\left|\nabla \rho_{h}-\nabla I_{h}(\rho(h))\right|+\left|\nabla I_{h}(\rho(h))-\nabla \rho(h)\right| \leq C h\left|\omega_{h}\right| .
$$


Accordingly,

$$
|\nabla \rho(h)| \leq h\left|\omega_{h}\right|+\left|\nabla \rho_{h}\right|
$$

Replacing this last inequality in (36), we get

$$
\left\|\nabla \rho_{h}\right\|_{L^{4}(\Omega)} \leq C h^{1 / 2}\left|\omega_{h}\right|+\left|\nabla \rho_{h}\right|^{1 / 2}\left|\omega_{h}\right|^{1 / 2} .
$$

On the other hand, taking $\bar{\rho}_{h}=\omega_{h}$ in (27) we arrive at

$$
\left|\omega_{h}\right|^{2}=\left(\nabla \rho_{h}, \nabla \omega_{h}\right) \leq\left|\nabla \rho_{h}\right|\left|\nabla \omega_{h}\right|
$$

Using the inverse inequality ([5]) $\left|\nabla \omega_{h}\right| \leq C h^{-1}\left|\omega_{h}\right|$, one has

$$
\left|\omega_{h}\right| \leq C h^{-1}\left|\nabla \rho_{h}\right| \text {. }
$$

Combining estimates (37) and (38), one arrives at (28).

Now, we are in position to prove stability estimates for the scheme.

Lemma 11. Suppose $\boldsymbol{u}_{0} \in \boldsymbol{V}, \rho_{0} \in H^{2}(\Omega)$ and $\boldsymbol{f} \in L^{2}\left(0, T ; \boldsymbol{L}^{p}(\Omega)\right)$ with $p>1$. Then, the solution of the discrete scheme (22), (23)-(24) satisfies the following estimates:

$$
\begin{array}{lll}
\text { i) } \max _{0 \leq n \leq N}\left|\boldsymbol{u}_{h}^{n}\right| \leq C, & \text { ii) } \sum_{n=0}^{N} k\left\|\boldsymbol{u}_{h}^{n}\right\|^{2} \leq C, & \text { iii) } \sum_{n=0}^{N-1}\left|\boldsymbol{u}_{h}^{n+1}-\boldsymbol{u}_{h}^{n}\right|^{2} \leq C, \\
\text { iv) } \max _{0 \leq n \leq N}\left|\nabla \rho_{h}^{n}\right| \leq C, & \text { v) } \sum_{n=0}^{N} k\left\|\nabla \rho_{h}^{n}\right\|_{L^{4}(\Omega)}^{4} \leq C, & \text { vi }) \sum_{n=0}^{N-1}\left|\nabla\left(\rho_{h}^{n+1}-\rho_{h}^{n}\right)\right|^{2} \leq C,
\end{array}
$$

with $C>0$ depending only on $\left(\rho_{0}, \boldsymbol{u}_{0}, \boldsymbol{f}\right)$.

Notice that, although the discrete density does not have $H^{2}$-regularity, $\boldsymbol{v}$ ) implies that the discrete density conserves the $L^{4}\left(0, T ; W^{1,4}(\Omega)\right)$-regularity.

Proof. First, we obtain a priori estimates for the velocity $\left(\boldsymbol{u}_{h}^{n}\right)$. Taking $\overline{\boldsymbol{u}}_{h}=2 k \boldsymbol{u}_{h}^{n+1}$ and $\bar{p}_{h}=p_{h}^{n+1}$ in (23)-(24), using the identity $(a-b, 2 a)=|a|^{2}-|b|^{2}+|a-b|^{2}$, using the properties (19) and (20) and taking into account Remark 7 , one has

$$
\begin{aligned}
& \left|\sqrt{\left[\rho_{h}^{n+1}\right]_{T}} \boldsymbol{u}_{h}^{n+1}\right|^{2}-\left|\sqrt{\left[\rho_{h}^{n}\right]_{T}} \boldsymbol{u}_{h}^{n}\right|^{2}+\left|\sqrt{\left[\rho_{h}^{n}\right]_{T}}\left(\boldsymbol{u}_{h}^{n+1}-\boldsymbol{u}_{h}^{n}\right)\right|^{2}+\mu_{1} k\left\|\boldsymbol{u}_{h}^{n+1}\right\|^{2} \\
& \leq 2 k\left(\left[\rho_{h}^{n+1}\right]_{T} \boldsymbol{f}^{n+1}, \boldsymbol{u}_{h}^{n+1}\right) \leq 2 k\left\|\left[\rho_{h}^{n+1}\right]_{T}\right\|_{L^{\infty}(\Omega)}\left\|\boldsymbol{f}^{n+1}\right\|_{L^{p}(\Omega)}\left\|\boldsymbol{u}_{h}^{n+1}\right\|_{L^{q}(\Omega)} \\
& \leq \frac{\mu_{1} k}{2}\left\|\boldsymbol{u}_{h}^{n+1}\right\|^{2}+C k\left\|\boldsymbol{f}^{n+1}\right\|_{L^{p}(\Omega)}^{2},
\end{aligned}
$$

where $q$ is the conjugate of $p$ such that $\frac{1}{p}+\frac{1}{q}=1$. Therefore,

$$
\begin{gathered}
\left|\sqrt{\left[\rho_{h}^{n+1}\right]_{T}} \boldsymbol{u}_{h}^{n+1}\right|^{2}-\left|\sqrt{\left[\rho_{h}^{n}\right]_{T}} \boldsymbol{u}_{h}^{n}\right|^{2}+\left|\sqrt{\left[\rho_{h}^{n}\right]_{T}}\left(\boldsymbol{u}_{h}^{n+1}-\boldsymbol{u}_{h}^{n}\right)\right|^{2}+\frac{\mu_{1}}{2} k\left\|\boldsymbol{u}_{h}^{n+1}\right\|^{2} \\
\leq C k\left\|\boldsymbol{f}^{n+1}\right\|_{L^{p}(\Omega)}^{2} .
\end{gathered}
$$

Adding (39) for $n=0, \ldots, r$ with $r<N$, the estimates for the velocity $\boldsymbol{i}$ ), $\boldsymbol{i i}$ ) and iii) hold.

To obtain a priori estimates of the discrete density, we write scheme (22) as (25). Taking $\bar{\omega}_{h}=2 k \omega_{h}^{n+1}$ in $(25) a$ and $\bar{\rho}_{h}=2\left(\rho_{h}^{n+1}-\rho_{h}^{n}\right)$ in $(25) b$, we arrive at:

$$
\left|\nabla \rho_{h}^{n+1}\right|^{2}-\left|\nabla \rho_{h}^{n}\right|^{2}+\left|\nabla\left(\rho_{h}^{n+1}-\rho_{h}^{n}\right)\right|^{2}+2 \lambda k\left|\omega_{h}^{n+1}\right|^{2}=-2 k\left(\boldsymbol{u}_{h}^{n} \cdot \nabla \rho_{h}^{n}, \omega_{h}^{n+1}\right) .
$$


Bounding the right-hand side term of (40) as

$$
\begin{aligned}
2 k\left|\left(\boldsymbol{u}_{h}^{n} \cdot \nabla \rho_{h}^{n}, \omega_{h}^{n+1}\right)\right| & \leq 2 k\left\|\boldsymbol{u}_{h}^{n}\right\|_{\mathbf{L}^{4}(\Omega)}\left\|\nabla \rho_{h}^{n}\right\|_{L^{4}(\Omega)}\left|\omega_{h}^{n+1}\right| \\
& \leq \lambda k\left|\omega_{h}^{n+1}\right|^{2}+\frac{k}{\lambda}\left\|\boldsymbol{u}_{h}^{n}\right\|_{L^{4}(\Omega)}^{2}\left\|\nabla \rho_{h}^{n}\right\|_{L^{4}(\Omega)}^{2},
\end{aligned}
$$

we get the inequality

$$
\left|\nabla \rho_{h}^{n+1}\right|^{2}-\left|\nabla \rho_{h}^{n}\right|^{2}+\left|\nabla\left(\rho_{h}^{n+1}-\rho_{h}^{n}\right)\right|^{2}+\lambda k\left|\omega_{h}^{n+1}\right|^{2} \leq \frac{k}{\lambda}\left\|\boldsymbol{u}_{h}^{n}\right\|_{L^{4}(\Omega)}^{2}\left\|\nabla \rho_{h}^{n}\right\|_{L^{4}(\Omega)}^{2} .
$$

Note that $\omega_{h}^{n}$ is defined for $n \geq 1$ in scheme (25). For $n=0$, it is enough to define $\omega_{h}^{0}$ as an approximation in $M_{h} \subset L_{0}^{2}(\Omega)$ of $-\Delta \rho_{0}$, and therefore to define $\rho_{h}^{0}$ based on $\omega_{h}^{0}$ using (25) $b$ jointly with the additional condition $\int_{\Omega} \rho_{h}^{0}=\int_{\Omega} \rho_{0}$. Of course, this initialization is possible in the case of initial density sufficiently regular, concretely $\rho_{0} \in H^{2}(\Omega)$. For the case $\rho_{0} \in H^{1}(\Omega)$, see Remark 14 below.

Using the discrete Gagliardo-Nirenberg inequality (28) in (41), one has

$\left|\nabla \rho_{h}^{n+1}\right|^{2}-\left|\nabla \rho_{h}^{n}\right|^{2}+\left|\nabla\left(\rho_{h}^{n+1}-\rho_{h}^{n}\right)\right|^{2}+\lambda k\left|\omega_{h}^{n+1}\right|^{2} \leq C k\left\|\boldsymbol{u}_{h}^{n}\right\|_{L^{4}(\Omega)}^{4}\left|\nabla \rho_{h}^{n}\right|^{2}+\frac{\lambda}{2} k\left|\omega_{h}^{n}\right|^{2}$.

Adding (42) for $n=0, \ldots, r$ with any $r<N$, we arrive at

$\left|\nabla \rho_{h}^{r+1}\right|^{2}+\lambda k \sum_{n=0}^{r}\left|\omega_{h}^{n+1}\right|^{2} \leq C \sum_{n=0}^{r} k\left\|\boldsymbol{u}_{h}^{n}\right\|_{L^{4}(\Omega)}^{4}\left|\nabla \rho_{h}^{n}\right|^{2}+\frac{\lambda}{2} k \sum_{n=0}^{r}\left|\omega_{h}^{n}\right|^{2}+\left|\nabla \rho_{h}^{0}\right|^{2}$.

Therefore,

$$
\left|\nabla \rho_{h}^{r+1}\right|^{2}+\frac{\lambda}{2} k \sum_{n=0}^{r}\left|\omega_{h}^{n+1}\right|^{2} \leq C \sum_{n=0}^{r} k\left\|\boldsymbol{u}_{h}^{n}\right\|_{L^{4}(\Omega)}^{4}\left|\nabla \rho_{h}^{n}\right|^{2}+\frac{\lambda}{2} k\left|\omega_{h}^{0}\right|^{2}+\left|\nabla \rho_{h}^{0}\right|^{2} .
$$

Applying the discrete Gronwall's lemma, using that

$$
k \sum_{n=0}^{N}\left\|\boldsymbol{u}_{h}^{n}\right\|_{L^{4}(\Omega)}^{4} \leq \max _{0 \leq n \leq N}\left|\boldsymbol{u}_{h}^{n}\right|^{2} k \sum_{n=0}^{N}\left\|\boldsymbol{u}_{h}^{n}\right\|^{2} \leq C
$$

(where $\boldsymbol{i}$ ) and $\boldsymbol{i i}$ ) are used) we obtain $\boldsymbol{i v}$ ), $\boldsymbol{v i}$ ) and

$$
k \sum_{n=0}^{N}\left|\omega_{h}^{n}\right|^{2} \leq C
$$

Finally, from (28) and estimates $i v$ ) and (44), one gets $\boldsymbol{v}$ ).

Remark 12. When in the discrete equation of density (22), the convection term is considered in a semi-implicit form, i.e. changing $\left(\boldsymbol{u}_{h}^{n} \cdot \nabla \rho_{h}^{n}, \bar{\rho}\right)$ by $\left(\boldsymbol{u}_{h}^{n} \cdot \nabla \rho_{h}^{n+1}, \bar{\rho}\right)$ in (22), then estimates for the discrete density $\boldsymbol{i v}$ ), $\boldsymbol{v}$ ) and $\boldsymbol{v} \boldsymbol{i}$ ) are obtained by imposing $C h / k^{2}<1$, which is independent of the regularity of the data. Indeed, we consider $C \sum_{n=0}^{r} k\left\|\boldsymbol{u}_{h}^{n}\right\|_{L^{4}(\Omega)}^{4}\left|\nabla \rho_{h}^{n+1}\right|^{2}$ instead of $C \sum_{n=0}^{r} k\left\|\boldsymbol{u}_{h}^{n}\right\|_{\mathbf{L}^{4}(\Omega)}^{4}\left|\nabla \rho_{h}^{n}\right|^{2}$ in (43). Then, in order to apply the generalised discrete Gronwall's lemma, we need to get that $C k\left\|\boldsymbol{u}_{h}^{n}\right\|_{L^{4}(\Omega)}^{4}<1$, which holds by using an inverse inequality,

$$
C k\left\|\boldsymbol{u}_{h}^{n}\right\|_{L^{4}(\Omega)}^{4} \leq C \frac{k}{h^{2}}\left\|\boldsymbol{u}_{h}^{n}\right\|_{L^{2}(\Omega)}^{4} \leq C \frac{k}{h^{2}}<1 .
$$


Remark 13. One also has the estimate $k \sum_{n=0}^{n}\left\|\rho_{h}^{n}\right\|_{W^{1, p}(\Omega)}^{2} \leq C$ for $p: 2<p<\infty$. For this, it suffices to see $\left\|\rho_{h}^{n}\right\|_{W^{1, p}(\Omega)} \leq C\left|\omega_{h}^{n}\right|$ and to apply (44). Indeed, $\left\|\rho_{h}^{n}\right\|_{W^{1, p}(\Omega)} \leq\left\|\rho_{h}^{n}-I_{h}(\rho(h))\right\|_{W^{1, p}(\Omega)}+\left\|I_{h}(\rho(h))-\rho(h)\right\|_{W^{1, p}(\Omega)}+\|\rho(h)\|_{W^{1, p}(\Omega)}$.

Using the inverse inequality $\left\|\bar{\omega}_{h}\right\|_{W^{1, p}(\Omega)} \leq C h^{-(p-2) / p}\left\|\bar{\omega}_{h}\right\|_{H^{1}(\Omega)}$ and (33), we bound

$$
\begin{aligned}
\left\|\rho_{h}^{n}-I_{h}(\rho(h))\right\|_{W^{1, p}(\Omega)} & \leq C h^{-(p-2) / p}\left\|\rho_{h}^{n}-I_{h}(\rho(h))\right\|_{H^{1}(\Omega)} \\
& \leq C h^{1-(p-2) / p}\left|\omega_{h}^{n}\right| \leq C\left|\omega_{h}^{n}\right|, \\
\left\|\rho(h)-I_{h}(\rho(h))\right\|_{W^{1, p}(\Omega)} & \leq C\|\rho(h)\|_{H^{2}(\Omega)} \leq C\left|\omega_{h}^{n}\right|, \\
\|\rho(h)\|_{W^{1, p}(\Omega)} & \leq C\|\rho(h)\|_{H^{2}(\Omega)} \leq\left|\omega_{h}^{n}\right| ;
\end{aligned}
$$

hence $\left\|\rho_{h}^{n}\right\|_{W^{1, p}(\Omega)} \leq C\left|\omega_{h}^{n}\right|$ holds.

Remark 14. Since $\left(\boldsymbol{u}_{0}, \rho_{0}\right) \in \boldsymbol{V} \times H^{2}(\Omega)$ has been imposed, then $\left\|\boldsymbol{u}_{h}^{0}\right\| \leq C\left\|\boldsymbol{u}_{0}\right\|$ and $\omega_{h}^{0}$ was defined based on $\Delta \rho_{0}$ and verifying $\left|\omega_{h}^{0}\right| \leq C\left|\Delta \rho_{0}\right|$. Imposing only $\left(\boldsymbol{u}_{0}, \rho_{0}\right) \in \boldsymbol{H} \times H^{1}(\Omega)$, the construction of $\omega_{h}^{0}$ must change. Firstly, we consider $\rho_{h}^{0} \in W_{h}$ as an approximation of $\rho_{0}$ in $H^{1}(\Omega)$ and, afterwards, we define $\omega_{h}^{0}$ from $(25)_{b}$. Therefore, using the inverse inequality $\left|\nabla \omega_{h}^{0}\right| \leq C h^{-1}\left|\omega_{h}^{0}\right|$ it is easy to prove that $\left|\omega_{h}^{0}\right| \leq C h^{-1}\left|\nabla \rho_{h}^{0}\right|$. Accordingly, to obtain the a priori estimates from (43), it is necessary to impose the constraint $k / h^{2} \leq C$ (since then, $\left.k\left|\omega_{h}^{0}\right|^{2} \leq C\left(k / h^{2}\right)\left|\nabla \rho_{h}^{0}\right|^{2} \leq C\left|\nabla \rho_{0}\right|\right)$. An analogous form is used to make the estimate $k\left\|\boldsymbol{u}_{h}^{0}\right\|_{L^{4}(\Omega)}^{2} \leq C\left(k / h^{2}\right)\left|\boldsymbol{u}_{h}^{0}\right|^{2} \leq C\left|\boldsymbol{u}_{0}\right|^{2}$, which is necessary to bound the first term of the sum $C k \sum_{n=0}^{r}\left\|\boldsymbol{u}_{h}^{n}\right\|_{L^{4}(\Omega)}^{4}\left|\nabla \rho_{h}^{n}\right|^{2}$.

Corollary 15 (Estimates for $\int_{\Omega} \rho_{h}^{n+1}$ ). It follows that

$$
\left|\int_{\Omega} \rho_{h}^{n+1}\right| \leq C
$$

where $C>0$ is a constant independent of $n$ and $h$.

Proof. Choosing $\bar{\rho}_{h}=1$ in (13), we get

$$
\int_{\Omega} \rho_{h}^{n+1}=\int_{\Omega} \rho_{h}^{n}-k \int_{\Omega} \boldsymbol{u}_{h}^{n} \cdot \nabla \rho_{h}^{n}
$$

Summing from $n=0, \ldots, r<N$, one has

$$
\int_{\Omega} \rho_{h}^{r+1}=\int_{\Omega} \rho_{h}^{0}-k \sum_{n=0}^{r} \int_{\Omega} \boldsymbol{u}_{h}^{n} \cdot \nabla \rho_{h}^{n} .
$$

Applying the Hölder inequality to the last term of the previous equality, this gives

$$
\left|\int_{\Omega} \rho_{h}^{r+1}\right| \leq\left|\int_{\Omega} \rho_{h}^{0}\right|+\left(k \sum_{n=0}^{r}\left|\boldsymbol{u}_{h}^{n}\right|^{2}\right)^{1 / 2}\left(k \sum_{n=0}^{r}\left|\nabla \rho_{h}^{n}\right|^{2}\right)^{1 / 2} \leq C,
$$

using estimates of Lemma 11.

Remark 16. If the density and pressure are approximated by the same space (i.e. $\left.W_{h} \cap L_{0}^{2}(\Omega)=M_{h}\right)$, then the average of the density is conserved, i.e. $\int_{\Omega} \rho_{h}^{n}=\int_{\Omega} \rho_{h}^{0}$, for each $n$ (this property is the discrete version of the continuous one $\int_{\Omega} \rho\left(\boldsymbol{x}, t_{1}\right) d \boldsymbol{x}=$ 
$\int_{\Omega} \rho\left(\boldsymbol{x}, t_{2}\right) d \boldsymbol{x}$ for any $t_{1}, t_{2} \in[0, T]$, whose physical meaning is the conservation of mass). To prove it, let us see first that $\left(\nabla \cdot \boldsymbol{u}_{h}^{n}, \bar{\rho}_{h}\right)=0$, for each $\bar{\rho}_{h} \in W_{h}$. Indeed, taking $\bar{\rho}_{h}-\frac{1}{|\Omega|} \int_{\Omega} \bar{\rho}_{h} \in M_{h}$ as a test function in (22), one has

$$
0=\left(\nabla \cdot \boldsymbol{u}_{h}^{n}, \bar{\rho}_{h}-\frac{1}{|\Omega|} \int_{\Omega} \bar{\rho}_{h}\right)=\left(\nabla \cdot \boldsymbol{u}_{h}^{n}, \bar{\rho}_{h}\right)-\frac{1}{|\Omega|} \int_{\Omega} \bar{\rho}_{h} \int_{\Omega} \nabla \cdot \boldsymbol{u}_{h}^{n}
$$

Since $\int_{\Omega} \nabla \cdot \boldsymbol{u}_{h}^{n}=0$, because $\boldsymbol{u}_{h}^{n}=0$ on $\partial \Omega$, one gets $\left(\nabla \cdot \boldsymbol{u}_{h}^{n}, \bar{\rho}_{h}\right)=0$. Therefore, if we choose $\bar{\rho}_{h}=1$ in (22) and apply that $\left(\boldsymbol{u}_{h}^{n} \cdot \nabla \rho_{h}^{n}, 1\right)=-\left(\nabla \cdot \boldsymbol{u}_{h}^{n}, \rho_{h}^{n}\right)=0$, one arrives at $\int_{\Omega} \rho_{h}^{n+1}=\int_{\Omega} \rho_{h}^{n}$, hence reasoning by induction $\int_{\Omega} \rho_{h}^{n}=\int_{\Omega} \rho_{h}^{0}$, for each $n$.

\section{WEAK CONVERGENCES}

In order to study the convergence of scheme (22), (23)-(24) towards the (unique) weak solution of (7), (9)-(10), we consider the following:

Definition 17. We define the auxiliary functions:

$$
\begin{gathered}
\boldsymbol{u}_{h, k}:[0, T] \rightarrow \boldsymbol{V}_{h} \text { such that } \boldsymbol{u}_{h, k}(t)=\boldsymbol{u}_{h}^{n+1}, t_{n} \leq t<t_{n+1}, \\
\widehat{\boldsymbol{u}}_{h, k}:[0, T] \rightarrow \boldsymbol{V}_{h} \text { such that } \widehat{\boldsymbol{u}}_{h, k}(t)=\boldsymbol{u}_{h}^{n}, t_{n} \leq t<t_{n+1}, \\
\rho_{h, k}:[0, T] \rightarrow W_{h} \text { such that } \rho_{h, k}(t)=\rho_{h}^{n+1}, t_{n} \leq t<t_{n+1}, \\
\widehat{\rho}_{h, k}:[0, T] \rightarrow W_{h} \text { such that } \widehat{\rho}_{h, k}(t)=\rho_{h}^{n}, t_{n} \leq t<t_{n+1}, \\
p_{h, k}:[0, T] \rightarrow M_{h} \text { such that } p_{h, k}(t)=p_{h}^{n+1}, t_{n} \leq t<t_{n+1}, \\
\widetilde{\rho}_{h, k}:[0, T] \rightarrow W_{h} \text { such that } \\
\widetilde{\rho}_{h, k}(t)=\rho_{h}^{n+1}+\frac{\rho_{h}^{n+1}-\rho_{h}^{n}}{k}\left(t-t_{n+1}\right), t_{n} \leq t<t_{n+1}, \\
w_{h, k}:[0, T] \rightarrow W_{h} \text { such that } w_{h, k}(t)=w_{h}^{n+1}, t_{n} \leq t<t_{n+1} .
\end{gathered}
$$

Using Lemma 11. Corollary 15 and the generalized Poincaré inequality

$$
\|\rho\|_{H^{1}(\Omega)} \leq C\left(|\nabla \rho|+\left|\int_{\Omega} \rho\right|\right)
$$

we arrive at the following:

Lemma 18. The following estimates (independent of $h$ and $k$ ) hold:

$$
\begin{aligned}
\left\{\boldsymbol{u}_{h, k}\right\}_{h, k},\left\{\widehat{\boldsymbol{u}}_{h, k}\right\}_{h, k} \quad \text { in } & L^{\infty}\left(0, T ; \boldsymbol{L}^{2}(\Omega)\right) \cap L^{2}\left(0, T ; \boldsymbol{H}_{0}^{1}(\Omega)\right), \\
\left\{\rho_{h, k}\right\}_{h, k},\left\{\widehat{\rho}_{h, k}\right\}_{h, k} \quad \text { in } & L^{\infty}\left(0, T ; H^{1}(\Omega)\right) \cap L^{4}\left(0, T ; W^{1,4}(\Omega)\right), \\
\left\{w_{h, k}\right\}_{h, k} \quad \text { in } & L^{2}\left(0, T ; L^{2}(\Omega)\right) .
\end{aligned}
$$

Moreover,

$$
\left\|\boldsymbol{u}_{h, k}-\widehat{\boldsymbol{u}}_{h, k}\right\|_{L^{2}\left(0, T ; L^{2}(\Omega)\right)}^{2} \leq C k \quad \text { and } \quad\left\|\rho_{h, k}-\widehat{\rho}_{h, k}\right\|_{L^{2}\left(0, T ; H^{1}(\Omega)\right)}^{2} \leq C k .
$$

Taking into account estimates (46)-(47) given in Lemma 18, there exist subsequences (denoted in the same way) with the corresponding weak convergences towards limit functions $\boldsymbol{u}, \hat{\boldsymbol{u}}, \rho, \hat{\rho}$. Moreover, thanks to (49), the identities of the limits $\boldsymbol{u}=\widehat{\boldsymbol{u}}$ and $\rho=\widehat{\rho}$ hold. 
Lemma 19. There exist subsequences of $\left\{\boldsymbol{u}_{h, k}\right\}_{h, k},\left\{\widehat{\boldsymbol{u}}_{h, k}\right\}_{h, k},\left\{\rho_{h, k}\right\}_{h, k},\left\{\widehat{\rho}_{h, k}\right\}_{h, k}$ (denoted in the same way) and limit functions $\boldsymbol{u}, \rho$ verifying the following weak convergences as $(h, k) \rightarrow 0$ :

$$
\begin{array}{r}
\boldsymbol{u}_{h, k} \rightarrow \boldsymbol{u} \text { and } \widehat{\boldsymbol{u}}_{h, k} \rightarrow \boldsymbol{u} \text { in }\left\{\begin{array}{l}
L^{2}\left(0, T ; \boldsymbol{H}_{0}^{1}(\Omega)\right) \text {-weak }, \\
L^{\infty}\left(0, T ; \boldsymbol{L}^{2}(\Omega)\right) \text {-weak },
\end{array}\right. \\
\rho_{h, k} \rightarrow \rho \text { and } \widehat{\rho}_{h, k} \rightarrow \rho \text { in }\left\{\begin{array}{l}
L^{4}\left(0, T ; W^{1,4}(\Omega)\right) \text {-weak }, \\
L^{\infty}\left(0, T ; H^{1}(\Omega)\right) \text {-weak },
\end{array}\right. \\
w_{h, k} \rightarrow w \text { in } L^{2}\left(0, T ; L^{2}(\Omega)\right) \text {-weak. }
\end{array}
$$

\section{Strong COnVERGences}

As usual in this type of nonlinear system, to obtain the convergence of the scheme we must get strong convergence for the approximations in some suitable space in order to identify the limit of the nonlinear terms.

\subsection{Strong Convergence for the density in $L^{2}(\Omega)$.}

Lemma 20.

$$
k \sum_{n=0}^{N}\left|\frac{\rho_{h}^{n+1}-\rho_{h}^{n}}{k}\right|^{2} \leq C,
$$

where $C>0$ depends only on $\left(\rho_{0}, \boldsymbol{u}_{0}, \boldsymbol{f}\right)$.

Proof. Taking $\bar{\rho}_{h}=\frac{\rho_{h}^{n+1}-\rho_{h}^{n}}{k}$ in (22) and using the identity $(a-b, 2 a)=|a|^{2}-$ $|b|^{2}+|a-b|^{2}$ we arrive at

$$
\begin{gathered}
\left|\frac{\rho_{h}^{n+1}-\rho_{h}^{n}}{k}\right|^{2}+\frac{\lambda}{2 k}\left(\left|\nabla \rho_{h}^{n+1}\right|^{2}-\left|\nabla \rho_{h}^{n}\right|^{2}+\left|\nabla \rho_{h}^{n+1}-\nabla \rho_{h}^{n}\right|^{2}\right) \\
=-\left(\boldsymbol{u}_{h}^{n} \cdot \nabla \rho_{h}^{n}, \frac{\rho_{h}^{n+1}-\rho_{h}^{n}}{k}\right) .
\end{gathered}
$$

We estimate the right-hand side as follows:

$$
\left(\boldsymbol{u}_{h}^{n} \cdot \nabla \rho_{h}^{n+1}, \frac{\rho_{h}^{n+1}-\rho_{h}^{n}}{k}\right) \leq \frac{1}{2}\left\|\boldsymbol{u}_{h}^{n}\right\|_{L^{4}(\Omega)}^{2}\left\|\nabla \rho_{h}^{n}\right\|_{L^{4}(\Omega)}^{2}+\frac{1}{2}\left|\frac{\rho_{h}^{n+1}-\rho_{h}^{n}}{k}\right|^{2} .
$$

Multiplying (50) by $2 k$, incorporating (51) and summing for $n=0, \ldots, N-1$ one gets

$$
k \sum_{n=0}^{N-1}\left|\frac{\rho_{h}^{n+1}-\rho_{h}^{n}}{k}\right|^{2}+\lambda\left|\nabla \rho_{h}^{N}\right|^{2} \leq k \sum_{n=0}^{N-1}\left\|\boldsymbol{u}_{h}^{n}\right\|_{L^{4}(\Omega)}^{2}\left\|\nabla \rho_{h}^{n}\right\|_{L^{4}(\Omega)}^{2}+\lambda\left|\nabla \rho_{h}^{0}\right|^{2},
$$

where

$$
k \sum_{n=0}^{N-1}\left\|\boldsymbol{u}_{h}^{n}\right\|_{L^{4}(\Omega)}^{2}\left\|\nabla \rho_{h}^{n}\right\|_{L^{4}(\Omega)}^{2} \leq \frac{1}{2}\left(k \sum_{n=0}^{N-1}\left\|\boldsymbol{u}_{h}^{n}\right\|_{L^{4}(\Omega)}^{4}+k \sum_{n=0}^{N-1}\left\|\nabla \rho_{h}^{n}\right\|_{L^{4}(\Omega)}^{4}\right) \leq C,
$$

thanks to the estimates from Lemma 11

Remark 21. As a consequence of the previous corollary, one has

$$
\left\|\frac{d}{d t} \widetilde{\rho}_{h, k}\right\|_{L^{2}\left(0, T ; L^{2}(\Omega)\right)} \leq C .
$$


On the other hand, by Lemma 11] $\left\|\widetilde{\rho}_{h, k}\right\|_{L^{4}\left(0, T ; W^{1,4}(\Omega)\right)} \leq C$ holds. Then, applying a compactness theorem of Aubin-Lions type,

$$
\widetilde{\rho}_{h, k} \rightarrow \rho \text { in } L^{4}\left(0, T ; L^{p}(\Omega)\right) \text { as }(h, k) \rightarrow 0, \quad \text { with } 1 \leq p<\infty .
$$

From this convergence we deduce that

$$
\rho_{h, k}, \widehat{\rho}_{h, k} \rightarrow \rho \text { in } L^{2}\left(0, T ; L^{2}(\Omega)\right) \text { as }(h, k) \rightarrow 0,
$$

using that

$$
\left\|\widetilde{\rho}_{h, k}-\rho_{k, h}\right\|_{L^{2}\left(0, T ; L^{2}(\Omega)\right)}^{2} \leq\left\|\widehat{\rho}_{h, k}-\rho_{k, h}\right\|_{L^{2}\left(0, T ; L^{2}(\Omega)\right)}^{2}=k \sum_{n=0}^{N-1}\left|\rho_{h}^{n+1}-\rho_{h}^{n}\right|^{2} \leq C k .
$$

Corollary 22. It follows that

$$
k \sum_{n=0}^{N}\left|\frac{\left[\rho_{h}^{n+1}\right]_{T}-\left[\rho_{h}^{n}\right]_{T}}{k}\right|^{2} \leq C .
$$

Proof. Using Lemma 20, it suffices to prove that $\left|\left[\rho_{h}^{n+1}(\boldsymbol{x}, t)\right]_{T}-\left[\rho_{h}^{n}(\boldsymbol{x}, t)\right]_{T}\right| \leq$ $\left|\rho_{h}^{n+1}(\boldsymbol{x}, t)-\rho_{h}^{n}(\boldsymbol{x}, t)\right|$ pointwise in $Q$ (here $|\cdot|$ denotes the absolute value function). But, this pointwise estimate is easy to verify taking into account that the approximations for the density are finite elements of degree 1.

5.2. Strong convergence for the density in $H^{1}(\Omega)$. Using the compactness of the discrete density in $L^{2}\left(0, T ; L^{2}(\Omega)\right)$, we are going to improve the strong convergence for the discrete density to the $L^{2}\left(0, T ; H^{1}(\Omega)\right)$ space. For this, we firstly have to identify $w=-\Delta \rho$. Indeed, let $\eta \in C_{c}^{\infty}(Q)$. We consider $\eta_{h}^{n}$ as the interpolated function of $\eta\left(t_{n}\right)$ in $W_{h}$ and define $\eta_{h, k} \in L^{\infty}\left(0, T ; W_{h}\right)$ as the piecewise constant function taking values $\eta_{h}^{n+1}$ in $\left(t_{n}, t_{n+1}\right]$. Then $\eta_{h, k} \rightarrow \eta$ in $L^{\infty}\left(0, T ; H^{1}(\Omega)\right)$ strongly as $(h, k) \rightarrow 0$. Therefore, setting $\bar{\rho}_{h}=\eta_{h}^{n+1}$ in (25) $b$, multiplying by $k$, summing over $n$ and doing $(h, k) \rightarrow 0$, we get

$$
\int_{Q}(\nabla \rho, \nabla \eta) \leftarrow \int_{Q}\left(\nabla \rho_{h, k}, \nabla \eta_{h, k}\right)=\int_{Q}\left(w_{h, k}, \eta_{h, k}\right) \rightarrow \int_{Q}(w, \eta) .
$$

Therefore, it is clear that $w=-\Delta \rho$ in $L^{2}(Q)$, and consequently $\rho \in L^{2}\left(0, T ; H^{2}(\Omega)\right)$.

Next, taking $\eta \in C^{\infty}(Q)$ and proceeding in the same manner, we recover the boundary condition $\frac{\partial \rho}{\partial \boldsymbol{n}}=0$ on $\Sigma$.

Corollary 23. One has that $\left\|\rho_{h, k}-\rho\right\|_{L^{2}\left(0, T ; H^{1}(\Omega)\right)} \rightarrow 0$ as $(h, k) \rightarrow 0$.

Proof. Considering $\bar{\rho}_{h}=\rho_{h}^{n+1}$ in (25) $b$, multiplying by $k$ and summing over $n$, one has

$$
\int_{0}^{T}\left|\nabla \rho_{h, k}\right|^{2}=\int_{0}^{T}\left(w_{h, k}, \rho_{h, k}\right) \rightarrow-\int_{0}^{T}(\Delta \rho, \rho)=\int_{0}^{T}|\nabla \rho|^{2} \quad \text { as }(h, k) \rightarrow 0
$$

because of $\left\{\rho_{h, k}\right\} \rightarrow \rho$ strongly in $L^{2}\left(0, T ; L^{2}(\Omega)\right)$ and $\left\{w_{h, k}\right\} \rightarrow-\Delta \rho$ weakly in $L^{2}\left(0, T ; L^{2}(\Omega)\right)$. Therefore, since $\left\|\rho_{h, k}\right\|_{L^{2}(Q)} \rightarrow\|\rho\|_{L^{2}(Q)}$ as $(h, k) \rightarrow 0$ by Remark 21 we have obtained that $\left\|\rho_{h, k}\right\|_{L^{2}\left(0, T, H^{1}(\Omega)\right.} \rightarrow\|\rho\|_{L^{2}\left(0, T ; H^{1}(\Omega)\right)}$ as $(h, k) \rightarrow 0$. Finally, from $\rho_{h, k} \rightarrow \rho$ weakly in $L^{2}\left(0, T ; H^{1}(\Omega)\right)$ by Lemma 19, we infer the desired result.

Taking into account estimate $\boldsymbol{i v}$ ) of Lemma 11, it is easy to check that

$$
\widehat{\rho}_{h, k} \rightarrow \rho \text { in } L^{2}\left(0, T ; H^{1}(\Omega)\right) \text { as }(h, k) \rightarrow 0 .
$$


5.3. Convergence for the density scheme. At this point, we study the convergence (as $(h, k) \rightarrow 0)$ for the incompressible condition and for the density scheme.

Proposition 24. The limit function $\boldsymbol{u}$ satisfies

$$
\nabla \cdot \boldsymbol{u}=0 \text { in } \Omega \times(0, T) .
$$

Proof. Let $q \in H^{1}\left(0, T ; H^{1}(\Omega)\right)$ be such that $\int_{\Omega} q(x) d \boldsymbol{x}=0$. We define $q_{h}^{n}$ as the interpolated into $M_{h}$ of $q\left(t_{n}\right)$ and by $q_{h, k} \in L^{\infty}\left(0, T ; M_{h}\right)$ the piecewise constant function taking values $q_{h}^{n+1}$ on $\left(t_{n}, t_{n+1}\right]$. Then, one has

$$
q_{k, h} \rightarrow q \quad \text { in } L^{\infty}\left(0, T ; L^{2}(\Omega)\right) .
$$

Taking $p_{h}^{n+1}=q_{h}^{n+1}$ as a test function in (24), multiplying by $k$ and adding for $n$,

$$
\int_{0}^{T}\left(\nabla \cdot \boldsymbol{u}_{h, k}, q_{h, k}\right) d t=0 .
$$

Thus, taking the limit in (53) as $(h, k) \rightarrow 0$ and using that

$$
\nabla \cdot \boldsymbol{u}_{h, k} \rightarrow \nabla \cdot \boldsymbol{u} \quad \text { in } L^{2}\left(0, T ; L^{2}(\Omega)\right) \text {-weak, as }(h, k) \rightarrow 0,
$$

one arrives at

$$
0=\lim _{(h, k) \rightarrow 0} \int_{0}^{T}\left(\nabla \cdot \boldsymbol{u}_{h, k}, q_{h, k}\right) d t=\int_{0}^{T}(\nabla \cdot \boldsymbol{u}, q) d t .
$$

Consequently, $\nabla \cdot \boldsymbol{u}=0$ holds in $Q$.

Proposition 25. The limit function $\rho \in L^{\infty}\left(0, T ; H_{N}^{1}(\Omega)\right) \cap L^{2}\left(0, T ; H_{N}^{2}(\Omega)\right)$ satisfies:

$$
\rho_{t}+\boldsymbol{u} \cdot \nabla \rho-\lambda \Delta \rho=0 \quad \text { a.e. in } Q
$$

and the pointwise estimate

$$
0<m \leq \rho(\boldsymbol{x}, t) \leq M<\infty \quad \text { in } Q .
$$

Proof. Let $\eta \in C_{c}^{\infty}(Q)$. We define $\eta_{h}^{n}$ as the interpolated function of $\eta\left(t_{n}\right)$ into $W_{h}$. We define $\eta_{h, k} \in L^{\infty}\left(0, T ; W_{h}\right)$ as the piecewise constant function taking values $\eta_{h}^{n+1}$ on $\left(t_{n}, t_{n+1}\right]$. One can also prove the following strong convergences as $(h, k) \rightarrow 0:$

$$
\eta_{k, h} \rightarrow \eta \quad \text { in } L^{\infty}\left(0, T ; H^{1}(\Omega)\right) .
$$

Taking $\bar{\rho}_{h}=\eta_{h}^{n+1}$ as a test function in (22), multiplying by $k$ and summing over $n$, we arrive at

$$
\begin{gathered}
-\int_{0}^{T}\left(\frac{d}{d t} \rho_{h, k}, \eta_{h, k}\right) d t+\int_{0}^{T}\left(\widehat{\boldsymbol{u}}_{h, k} \cdot \nabla \widehat{\rho}_{h, k}, \eta_{h, k}\right) d t \\
+\lambda \int_{0}^{T}\left(\nabla \rho_{h, k}, \nabla \eta_{h, k}\right) d t=0 .
\end{gathered}
$$

Taking the limit as $(h, k) \rightarrow 0$ in (56) and using the following convergences as $(h, k) \rightarrow 0$ :

- $\widehat{\boldsymbol{u}}_{h, k} \rightarrow \boldsymbol{u}$ in $L^{2}\left(0, T ; \boldsymbol{H}_{0}^{1}(\Omega)\right)$-weak,

- $\widehat{\rho}_{h, k} \rightarrow \rho$ in $L^{2}\left(0, T ; H^{1}(\Omega)\right)$-strong, $\frac{d}{d t} \rho_{h, k} \rightarrow \frac{d}{d t} \rho$ in $L^{2}\left(0, T ; L^{2}(\Omega)\right)$ weak,

the proof is concluded by using the additional regularity $\rho \in L^{2}\left(0, T ; H^{2}(\Omega)\right)$. 
5.4. Strong Convergence in $L^{2}(\Omega)$ for the truncated density. Using Proposition 25, we establish the following compactness result.

\section{Proposition 26.}

$$
\left[\rho_{h, k}\right]_{T},\left[\widehat{\rho}_{h, k}\right]_{T} \rightarrow \rho \text { in } L^{2}\left(0, T ; L^{2}(\Omega)\right) \text {-strong, as }(h, k) \rightarrow 0 .
$$

Proof. We will show the proof for $\rho_{h, k}$, and analogously it can be shown for $\widehat{\rho}_{h, k}$. We define the following pointwise truncating operator:

$$
T_{m}^{M} \rho_{h, k}(\boldsymbol{x}, t)=\left\{\begin{array}{lll}
\rho_{h, k}(\boldsymbol{x}, t) & \text { if } & \rho_{h, k}(\boldsymbol{x}, t) \in[m, M], \\
m & \text { if } & \rho_{h, k}(\boldsymbol{x}, t)<m, \\
M & \text { if } & \rho_{h, k}(\boldsymbol{x}, t)>M .
\end{array}\right.
$$

Notice that, in general, $T_{m}^{M} \rho_{h, k} \notin W_{h}$. Let us see first that $T_{m}^{M} \rho_{h, k} \rightarrow \rho$ in $L^{2}\left(0, T ; L^{2}(\Omega)\right)$-strong as $(h, k) \rightarrow 0$. Indeed, since $\rho_{h, k} \rightarrow \rho$ in $L^{2}\left(0, T ; L^{2}(\Omega)\right)$ strong, as $(h, k) \rightarrow 0$, one can extract a subsequence (denoted in the same way) such that:

$$
\rho_{h, k}(\boldsymbol{x}, t) \rightarrow \rho(\boldsymbol{x}, t) \text { a.e. }(\boldsymbol{x}, t) \in Q, \quad \text { as }(h, k) \rightarrow 0 .
$$

Therefore, if we consider $(\boldsymbol{x}, t) \in Q$ such that $\rho(\boldsymbol{x}, t) \in(m, M)$, then there exist $h_{0}(\boldsymbol{x}, t)>0$ and $k_{0}(\boldsymbol{x}, t)>0$ so that $\rho_{h, k}(\boldsymbol{x}, t) \in(m, M)$ holds for all $h \leq h_{0}(\boldsymbol{x}, t)$ and $k \leq k_{0}(\boldsymbol{x}, t)$. Thus, $\rho_{h, k}(\boldsymbol{x}, t)=T_{m}^{M} \rho_{h, k}(\boldsymbol{x}, t)$ and $T_{m}^{M} \rho_{h, k}(\boldsymbol{x}, t) \rightarrow \rho(\boldsymbol{x}, t)$ as $(h, k) \rightarrow 0$.

On the other hand, if $(\boldsymbol{x}, t) \in Q$ is such that $\rho(\boldsymbol{x}, t)=m$, then

$$
\begin{aligned}
& \forall \varepsilon>0, \exists\left(h_{0}, k_{0}\right)(\boldsymbol{x}, t) \text { such that } \forall(h, k) \leq\left(h_{0}, k_{0}\right)(\boldsymbol{x}, t), \text { one has } \\
& \left|\rho_{h, k}(\boldsymbol{x}, t)-m\right|=\left|\rho_{h, k}(\boldsymbol{x}, t)-\rho(\boldsymbol{x}, t)\right|<\varepsilon .
\end{aligned}
$$

If we choose $\varepsilon<M-m$ this gives $T_{m}^{M} \rho_{h, k}(\boldsymbol{x}, t)=\rho_{h, k}(\boldsymbol{x}, t)$ or $T_{m}^{M} \rho_{h, k}(\boldsymbol{x}, t)=m$. Therefore, $\forall \varepsilon>0$ (with $\varepsilon<M-m$ ) and $\forall(h, k) \leq\left(h_{0}, k_{0}\right)(\boldsymbol{x}, t)$ one has

$$
\left|T_{m}^{M} \rho_{h, k}(\boldsymbol{x}, t)-\rho(\boldsymbol{x}, t)\right|<\varepsilon \text {. }
$$

Finally, the remaining case of $(\boldsymbol{x}, t) \in Q$ such that $\rho(\boldsymbol{x}, t)=M$ is proved in the same manner.

Consequently, we have that $T_{m}^{M} \rho_{h, k}(\boldsymbol{x}, t) \rightarrow \rho(\boldsymbol{x}, t)$ a.e. $(\boldsymbol{x}, t) \in Q$ as $(h, k) \rightarrow$ 0 . Therefore, using that $\left|T_{m}^{M} \rho_{h, k}(\boldsymbol{x}, t)\right| \leq M$ a.e. $(\boldsymbol{x}, t) \in Q$, we can apply the Dominated Convergence Theorem, obtaining

$$
T_{m}^{M} \rho_{h, k} \rightarrow \rho \text { in } L^{2}\left(0, T ; L^{2}(\Omega)\right) \text {-strong, as }(h, k) \rightarrow 0 .
$$

Using the interpolation operator $I_{h}$ from $H^{1}(\Omega) \cap c^{0}(\bar{\Omega})$ into $W_{h}$, it is easy to check that

hence we can write

$$
\left[\rho_{h, k}\right]_{T}=I_{h}\left(T_{m}^{M} \rho_{h, k}\right)
$$

$$
\left[\rho_{h, k}\right]_{T}-\rho=I_{h}\left(T_{m}^{M} \rho_{h, k}\right)-I_{h}(\rho)+I_{h}(\rho)-\rho .
$$

It is verified that

$$
I_{h}\left(T_{m}^{M} \rho_{h, k}\right)-I_{h}(\rho) \rightarrow 0 \text { in } L^{2}\left(0, T ; L^{2}(\Omega)\right) .
$$

Indeed, taking the norm in $L^{2}(\Omega)$,

$$
\begin{aligned}
\left|I_{h}\left(T_{m}^{M} \rho_{h, k}\right)-I_{h}(\rho)\right| & =\left|I_{h}\left(T_{m}^{M} \rho_{h, k}-\rho\right)\right| \\
& \leq\left|I_{h}\left(T_{m}^{M} \rho_{h, k}-\rho\right)-\left(T_{m}^{M} \rho_{h, k}-\rho\right)\right|+\left|T_{m}^{M} \rho_{h, k}-\rho\right| \\
& \leq C h\left\|T_{m}^{M} \rho_{h, k}-\rho\right\|_{H^{1}(\Omega)}+\left|T_{m}^{M} \rho_{h, k}-\rho\right| .
\end{aligned}
$$


Next, taking the norm in $L^{2}(0, T)$,

$$
\begin{array}{rl}
\left\|I_{h}\left(T_{m}^{M} \rho_{h, k}\right)-I_{h}(\rho)\right\|_{L^{2}\left(0, T ; L^{2}(\Omega)\right)} \leq C & h\left\|T_{m}^{M} \rho_{h, k}-\rho\right\|_{L^{2}\left(0, T ; H^{1}(\Omega)\right)} \\
& +\left\|T_{m}^{M} \rho_{h, k}-\rho\right\|_{L^{2}\left(0, T ; L^{2}(\Omega)\right)} .
\end{array}
$$

Therefore, using (58) and that $T_{m}^{M} \rho_{h, k}$ is bounded in $L^{2}\left(0, T ; H^{1}(\Omega)\right.$ ) (thanks to $\left|\nabla T_{m}^{M} \rho_{h, k}\right| \leq\left|\nabla \rho_{h, k}\right|$ and that $\left\{\rho_{h, k}\right\}_{h, k}$ is bounded in $\left.L^{2}\left(0, T ; H^{1}(\Omega)\right)\right)$, (60) holds.

As well $I_{h}(\rho)-\rho \rightarrow 0$ in $L^{2}\left(0, T ; L^{2}(\Omega)\right)$, thanks to $\left|I_{h}(\rho)-\rho\right| \leq C h\|\rho\|_{H^{1}(\Omega)}$; hence

$$
\left\|I_{h}(\rho)-\rho\right\|_{L^{2}\left(0, T ; L^{2}(\Omega)\right)} \leq C h\|\rho\|_{L^{2}\left(0, T ; H^{1}(\Omega)\right)} .
$$

From (59), we arrive at (57).

\subsection{Strong convergence for the velocity.}

Proposition 27. The following "fractional in time" estimate holds:

$$
\int_{0}^{T-\delta}\left|\sqrt{\left[\rho_{h, k}\right]_{T}(t+\delta)}\left(\boldsymbol{u}_{h, k}(t+\delta)-\boldsymbol{u}_{h, k}(t)\right)\right|^{2} d t \leq C \delta^{1 / 2} \quad \forall \delta: \quad 0<\delta<T,
$$

with $C>0$ independent of $h, k$ and $\delta$.

Proof. Since $\rho_{h, k}$ and $\boldsymbol{u}_{h, k}$ are piecewise constant functions, to obtain (61) it suffices to consider $\delta$ as a multiple of the time step $k$, that is, $\delta=r k$ with $r=0, \ldots, N$ and to prove

$$
k \sum_{m=0}^{N-r}\left|\sqrt{\left[\rho_{h}^{m+r}\right]_{T}}\left(\boldsymbol{u}_{h}^{m+r}-\boldsymbol{u}_{h}^{m}\right)\right|^{2} \leq C(r k)^{1 / 2}, \quad \forall r: 0 \leq r \leq N .
$$

Firstly, we will write the time derivative to the momentum equation (23) in a conservative form. It is obtained by adding to both sides of (23) the term

$$
\frac{1}{2}\left(\frac{\left[\rho_{h}^{n+1}\right]_{T}-\left[\rho_{h}^{n}\right]_{T}}{k}, \boldsymbol{u}_{h}^{n+1} \cdot \overline{\boldsymbol{u}}_{h}\right)
$$

as follows:

$$
\left\{\begin{array}{l}
\left(\frac{\left[\rho_{h}^{n+1}\right]_{T} \boldsymbol{u}_{h}^{n+1}-\left[\rho_{h}^{n}\right]_{T} \boldsymbol{u}_{h}^{n}}{k}, \overline{\boldsymbol{u}}_{h}\right)+a\left(\left[\rho_{h}^{n+1}\right]_{T}, \boldsymbol{u}_{h}^{n+1}, \overline{\boldsymbol{u}}_{h}\right) \\
+c\left(\rho_{h}^{n+1} \boldsymbol{u}_{h}^{n}-\lambda \nabla \rho_{h}^{n+1}, \boldsymbol{u}_{h}^{n+1}, \overline{\boldsymbol{u}}_{h}\right)-\left(p_{h}^{n+1}, \nabla \cdot \overline{\boldsymbol{u}}_{h}\right) \\
=\left(\left[\rho_{h}^{n+1}\right]_{T} \boldsymbol{f}^{n+1}, \overline{\boldsymbol{u}}_{h}\right)+\frac{1}{2}\left(\frac{\left[\rho_{h}^{n+1}\right]_{T}-\left[\rho_{h}^{n}\right]_{T}}{k}, \boldsymbol{u}_{h}^{n+1} \cdot \overline{\boldsymbol{u}}_{h}\right) .
\end{array}\right.
$$

Multiplying by $k$ and summing for $n=m, \ldots, m-1+r$ in (63), we arrive at (64)

$$
\left\{\begin{array}{l}
\left(\left[\rho_{h}^{m+r}\right]_{T} \boldsymbol{u}_{h}^{m+r}-\left[\rho_{h}^{m}\right]_{T} \boldsymbol{u}_{h}^{m}, \overline{\boldsymbol{u}}_{h}\right)+k \sum_{n=m}^{m-1+r} a\left(\left[\rho_{h}^{n+1}\right]_{T}, \boldsymbol{u}_{h}^{n+1}, \overline{\boldsymbol{u}}_{h}\right) \\
+k \sum_{n=m}^{m-1+r} c\left(\rho_{h}^{n+1} \boldsymbol{u}_{h}^{n}-\lambda \nabla \rho_{h}^{n+1}, \boldsymbol{u}_{h}^{n+1}, \overline{\boldsymbol{u}}_{h}\right)-\sum_{n=m}^{m-1+r}\left(p_{h}^{n+1}, \nabla \cdot \overline{\boldsymbol{u}}_{h}\right) \\
=k \sum_{n=m}^{m-1+r}\left(\left[\rho_{h}^{n+1}\right]_{T} \boldsymbol{f}^{n+1}, \overline{\boldsymbol{u}}_{h}\right)+\frac{k}{2} \sum_{n=m}^{m-1+r}\left(\frac{\left[\rho_{h}^{n+1}\right]_{T}-\left[\rho_{h}^{n}\right]_{T}}{k}, \boldsymbol{u}_{h}^{n+1} \cdot \overline{\boldsymbol{u}}_{h}\right) .
\end{array}\right.
$$


Taking $\overline{\boldsymbol{u}}_{h}=\boldsymbol{u}_{h}^{m+r}-\boldsymbol{u}_{h}^{m}$ as a test function and keeping in mind the identity

$$
\begin{array}{r}
\left(\left[\rho_{h}^{m+r}\right]_{T} \boldsymbol{u}_{h}^{m+r}-\left[\rho_{h}^{m}\right]_{T} \boldsymbol{u}_{h}^{m}, \boldsymbol{u}_{h}^{m+r}-\boldsymbol{u}_{h}^{m}\right)=\left(\left[\rho_{h}^{m+r}\right]_{T}\left(\boldsymbol{u}_{h}^{m+r}-\boldsymbol{u}_{h}^{m}\right), \boldsymbol{u}_{h}^{m+r}-\boldsymbol{u}_{h}^{m}\right) \\
+\left(\left[\rho_{h}^{m+r}\right]_{T}-\left[\rho_{h}^{m}\right]_{T}, \boldsymbol{u}_{h}^{m} \cdot\left(\boldsymbol{u}_{h}^{m+r}-\boldsymbol{u}_{h}^{m}\right)\right)
\end{array}
$$

we get

$$
\left\{\begin{array}{l}
\left|\sqrt{\left[\rho_{h}^{m+r}\right]_{T}}\left(\boldsymbol{u}_{h}^{m+r}-\boldsymbol{u}_{h}^{m}\right)\right|^{2}=-\left(\left[\rho_{h}^{m}\right]_{T}-\left[\rho_{h}^{m+r}\right]_{T}, \boldsymbol{u}_{h}^{m} \cdot\left(\boldsymbol{u}_{h}^{m+r}-\boldsymbol{u}_{h}^{m}\right)\right) \\
-k \sum_{n=m}^{m-1+r} a\left(\left[\rho_{h}^{n+1}\right]_{T}, \boldsymbol{u}_{h}^{n+1}, \boldsymbol{u}_{h}^{m+r}-\boldsymbol{u}_{h}^{m}\right) \\
-k \sum_{n=m}^{m-1+r} c\left(\rho_{h}^{n+1} \boldsymbol{u}_{h}^{n}-\lambda \nabla \rho_{h}^{n+1}, \boldsymbol{u}_{h}^{n+1}, \boldsymbol{u}_{h}^{m+r}-\boldsymbol{u}_{h}^{m}\right) \\
+k \sum_{n=m}^{m-1+r}\left(\left[\rho_{h}^{n+1}\right]_{T} \boldsymbol{f}^{n+1}, \boldsymbol{u}_{h}^{m+r}-\boldsymbol{u}_{h}^{m}\right) \\
+k \sum_{n=m}^{m-1+r} \frac{1}{2}\left(\frac{\left[\rho_{h}^{n+1}\right]_{T}-\left[\rho_{h}^{n}\right]_{T}}{k}, \boldsymbol{u}_{h}^{n+1} \cdot\left(\boldsymbol{u}_{h}^{m+r}-\boldsymbol{u}_{h}^{m}\right)\right) .
\end{array}\right.
$$

On the other hand, multiplying by $k$, summing for $n=m, \ldots, m-1+r$ and taking as a test function $\bar{\rho}_{h}=\rho_{h}^{m}-\rho_{h}^{m+r}$ in the density equation $(\underline{25}) a$, we find the equality

$$
\left|\rho_{h}^{m}-\rho_{h}^{m+r}\right|^{2}=-k \sum_{n=m}^{m-1+r}\left(\boldsymbol{u}_{h}^{n} \cdot \nabla \rho_{h}^{n}+\lambda \omega_{h}^{n+1}, \rho_{h}^{m}-\rho_{h}^{m+r}\right) .
$$

Now, estimating the right-hand side of (66) as

$$
\left|\rho_{h}^{m}-\rho_{h}^{m+r}\right|^{2} \leq k \sum_{n=m}^{m-1+r}\left(\left\|\boldsymbol{u}_{h}^{n}\right\|_{L^{4}(\Omega)}\left\|\nabla \rho_{h}^{n}\right\|_{L^{4}(\Omega)}+\lambda\left|\omega_{h}^{n+1}\right|\right)\left|\rho_{h}^{m}-\rho_{h}^{m+r}\right|,
$$

one gets

$$
\begin{aligned}
\left|\rho_{h}^{m}-\rho_{h}^{m+r}\right| & \leq C k \sum_{n=m}^{m-1+r}\left(\left\|\boldsymbol{u}_{h}^{n}\right\|_{L^{4}(\Omega)}\left\|\nabla \rho_{h}^{n}\right\|_{L^{4}(\Omega)}+\left|\omega_{h}^{n+1}\right|\right) \\
& \leq C k\left(\sum_{n=m}^{m-1+r}\left\|\boldsymbol{u}_{h}^{n}\right\|_{L^{4}(\Omega)}^{2}\left\|\nabla \rho_{h}^{n}\right\|_{L^{4}(\Omega)}^{2}+\left|\omega_{h}^{n+1}\right|^{2}\right)^{1 / 2}(r k)^{1 / 2} \\
& \leq C(r k)^{1 / 2} .
\end{aligned}
$$

Therefore, we have obtained that $\max _{1 \leq m \leq N}\left|\rho_{h}^{m}-\rho_{h}^{m+r}\right| \leq C(r k)^{1 / 2}$. Consequently, using that $\left|\left[\rho_{h}^{m}\right]_{T}-\left[\rho_{h}^{m+r}\right]_{T}\right| \leq\left|\rho_{h}^{m}-\rho_{h}^{m+r}\right|$, one also obtains that

$$
\max _{1 \leq m \leq N}\left|\left[\rho_{h}^{m}\right]_{T}-\left[\rho_{h}^{m+r}\right]_{T}\right| \leq C(r k)^{1 / 2}
$$

Multiplying by $k$ and summing for $m=0, \ldots, N-r$ in (65) and bounding adequately, we can obtain the required bound (62). For brevity, we only bound the two main terms of (65):

$$
-k \sum_{m=0}^{N-r}\left(\left[\rho_{h}^{m}\right]_{T}-\left[\rho_{h}^{m+r}\right]_{T}, \boldsymbol{u}_{h}^{m} \cdot\left(\boldsymbol{u}_{h}^{m+r}-\boldsymbol{u}_{h}^{m}\right)\right) \leq C r k
$$




$$
-k^{2} \sum_{m=0}^{N-r} \sum_{n=m}^{m-1+r} c\left(\rho_{h}^{n+1} \boldsymbol{u}_{h}^{n}-\lambda \nabla \rho_{h}^{n+1}, \boldsymbol{u}_{h}^{m+r}-\boldsymbol{u}_{h}^{m}, \boldsymbol{u}_{h}^{n+1}\right) \leq C(r k)^{1 / 2} .
$$

Indeed, we bound (68) as follows:

$$
\begin{aligned}
& -k \sum_{m=0}^{N-r}\left(\left[\rho_{h}^{m}\right]_{T}-\left[\rho_{h}^{m+r}\right]_{T}, \boldsymbol{u}_{h}^{m} \cdot\left(\boldsymbol{u}_{h}^{m+r}-\boldsymbol{u}_{h}^{m}\right)\right) \\
& \leq k \sum_{m=0}^{N-r}\left|\left[\rho_{h}^{m}\right]_{T}-\left[\rho_{h}^{m+r}\right]_{T}\right|\left\|\boldsymbol{u}_{h}^{m}\right\|_{L^{4}(\Omega)}\left\|\boldsymbol{u}_{h}^{m+r}-\boldsymbol{u}_{h}^{m}\right\|_{L^{4}(\Omega)} \\
& \leq \max _{1 \leq m \leq N}\left|\left[\rho_{h}^{m}\right]_{T}-\left[\rho_{h}^{m+r}\right]_{T}\right|\left(k \sum_{m=0}^{N-r}\left\|\boldsymbol{u}_{h}^{m}\right\|_{L^{4}(\Omega)}^{2}\right)^{1 / 2}\left(k \sum_{m=0}^{N-r}\left\|\boldsymbol{u}_{h}^{m+r}-\boldsymbol{u}_{h}^{m}\right\|_{L^{4}(\Omega)}\right)^{1 / 2} \\
& \leq C(r k)^{1 / 2} \quad \text { (using (67)). }
\end{aligned}
$$

We bound (69) using (21), as follows:

$$
\begin{aligned}
& k^{2} \sum_{m=0}^{N-r} \sum_{n=m}^{m-1+r} c\left(\rho_{h}^{n+1} \boldsymbol{u}_{h}^{n}-\lambda \nabla \rho_{h}^{n+1}, \boldsymbol{u}_{h}^{m+r}-\boldsymbol{u}_{h}^{m}, \boldsymbol{u}_{h}^{n+1}\right) \\
& \leq C k^{2} \sum_{m=0}^{N-r} \sum_{\substack{n=m \\
m-1+r}}^{N-r} \rho_{h}^{n+1} \boldsymbol{u}_{h}^{n}-\lambda \nabla \rho_{h}^{n+1}\left\|_{L^{3}}\right\| \boldsymbol{u}_{h}^{n+1}\|\| \boldsymbol{u}_{h}^{m+r}-\boldsymbol{u}_{h}^{m} \| \\
& \leq C k^{2} \sum_{m=0}^{N-r} \sum_{n=m}^{m-1+r}\left(\left\|\rho_{h}^{n+1}\right\|_{H^{1}(\Omega)}\left\|\boldsymbol{u}_{h}^{n}\right\|+\left|\omega_{h}^{n+1}\right|\right)\left\|\boldsymbol{u}_{h}^{n+1}\right\|\left\|\boldsymbol{u}_{h}^{m+r}-\boldsymbol{u}_{h}^{m}\right\| .
\end{aligned}
$$

Interchanging the sum order (Fubini's discrete rule) and using the estimate $\left\|\rho_{h}^{n+1}\right\|_{H^{1}(\Omega)} \leq C$, we get

$$
\begin{aligned}
& k^{2} \sum_{m=0}^{N-r} \sum_{n=m}^{m-1+r} c\left(\rho_{h}^{n+1} \boldsymbol{u}_{h}^{n}-\lambda \nabla \rho_{h}^{n+1}, \boldsymbol{u}_{h}^{m+r}-\boldsymbol{u}_{h}^{m}, \boldsymbol{u}_{h}^{n+1}\right) \\
& \leq C k^{2} \sum_{n=0}^{N-1}\left(\left\|\boldsymbol{u}_{h}^{n}\right\|+\left|\omega_{h}^{n+1}\right|\right)\left\|\boldsymbol{u}_{h}^{n+1}\right\| \sum_{m=\overline{n-r+1}}^{n}\left\|\boldsymbol{u}_{h}^{m+r}-\boldsymbol{u}_{h}^{m}\right\|,
\end{aligned}
$$

where

$$
\bar{n}=\left\{\begin{array}{lll}
0 & \text { if } \quad n<0 \\
n & \text { if } \quad 0 \leq n \leq N-r \\
N-r & \text { if } \quad n>N-r
\end{array}\right.
$$

Next, using the inequality $|\bar{n}-\overline{n-r+1}| \leq r$,

$$
\begin{aligned}
& k^{2} \sum_{m=0}^{N-r} \sum_{n=m}^{m-1+r} c\left(\rho_{h}^{n+1} \boldsymbol{u}_{h}^{n}-\lambda \nabla \rho_{h}^{n+1}, \boldsymbol{u}_{h}^{m+r}-\boldsymbol{u}_{h}^{m}, \boldsymbol{u}_{h}^{n+1}\right) \\
& \leq C \sum_{n=0}^{N-1} k\left(\left\|\boldsymbol{u}_{h}^{n}\right\|+\left|\omega_{h}^{n+1}\right|\right)\left\|\boldsymbol{u}_{h}^{n+1}\right\|\left(\sum_{m=\overline{n-r+1}}^{\bar{n}} k\left\|\boldsymbol{u}_{h}^{m+r}-\boldsymbol{u}_{h}^{m}\right\|^{2}\right)^{1 / 2}\left(\sum_{m=\overline{n-r+1}}^{\bar{n}} k\right)^{1 / 2} \\
& \leq C(r k)^{1 / 2}\left(k \sum_{n=0}^{N-1}\left(\left\|\boldsymbol{u}_{h}^{n}\right\|+\left|\omega_{h}^{n+1}\right|\right)^{2}\right)^{1 / 2}\left(k \sum_{n=0}^{N-1}\left\|\boldsymbol{u}_{h}^{n+1}\right\|^{2}\right)^{1 / 2} \leq C(r k)^{1 / 2},
\end{aligned}
$$

and the proof is finished. 
Remark 28. From the a priori estimates of $\boldsymbol{u}_{h, k}$ in $L^{\infty}\left(0, T ; \boldsymbol{L}^{2}(\Omega)\right) \cap L^{2}(0, T$; $\left.\boldsymbol{H}_{0}^{1}(\Omega)\right)$ and the fractional in time estimate (61), we can apply a compactness result ([16]) of Aubin-Lions type, obtaining that

$$
\boldsymbol{u}_{h, k} \rightarrow \boldsymbol{u} \text { in } L^{2}\left(0, T ; \boldsymbol{L}^{2}(\Omega)\right) \text {-strong as }(h, k) \rightarrow 0 .
$$

Consequently, thanks to (49),

$$
\widehat{\boldsymbol{u}}_{h, k} \rightarrow \boldsymbol{u} \text { in } L^{2}\left(0, T ; \boldsymbol{L}^{2}(\Omega)\right) \text {-strong as }(h, k) \rightarrow 0 .
$$

\section{Convergence for the momentum system}

In order to eliminate the discrete pressure, we are going to consider adequate test functions, thanks to the following:

Lemma 29. Let $\overline{\boldsymbol{u}} \in \boldsymbol{H}^{2}(\Omega) \cap \boldsymbol{H}_{0}^{1}(\Omega)$. Then, there exists $\overline{\boldsymbol{u}}_{h} \in \boldsymbol{V}_{h}$ such that:

$$
\begin{array}{ll}
\text { i) } & \overline{\boldsymbol{u}}_{h} \rightarrow \overline{\boldsymbol{u}} \text { in } \boldsymbol{H}_{0}^{1}(\Omega), \\
\text { ii) } & \left(\nabla \cdot \overline{\boldsymbol{u}}_{h}, q_{h}\right)=\left(\nabla \cdot \overline{\boldsymbol{u}}, q_{h}\right), \forall q_{h} \in M_{h} .
\end{array}
$$

Proof. We consider $\boldsymbol{t}_{h}$ as the interpolation of $\overline{\boldsymbol{u}}$ into $\mathbf{V}_{h}$. Then, $\left\|\overline{\boldsymbol{u}}-\boldsymbol{t}_{h}\right\| \rightarrow 0$ as $h \rightarrow 0$. We define $\left(\boldsymbol{e}_{h}, r_{h}\right) \in \mathbf{V}_{h} \times M_{h}$ as the solution of the following "discrete Stokes" problem:

$$
\left\{\begin{array}{l}
\left(\nabla \boldsymbol{e}_{h}, \nabla \boldsymbol{y}_{h}\right)-\left(r_{h}, \nabla \cdot \boldsymbol{y}_{h}\right)=\left(\nabla\left(\overline{\boldsymbol{u}}-\boldsymbol{t}_{h}\right), \nabla \boldsymbol{y}_{h}\right), \quad \forall \boldsymbol{y}_{h} \in \mathbf{V}_{h}, \\
\left(\nabla \cdot \boldsymbol{e}_{h}, \bar{p}_{h}\right)=\left(\nabla \cdot\left(\overline{\boldsymbol{u}}-\boldsymbol{t}_{h}\right), \bar{p}_{h}\right), \quad \forall \bar{p}_{h} \in M_{h} .
\end{array}\right.
$$

It is easy to deduce, using the inf-sup condition, that (70) has a unique solution. The estimate

$$
\left\|\boldsymbol{e}_{h}\right\|+\left|r_{h}\right| \leq C\left\|\overline{\boldsymbol{u}}-\boldsymbol{t}_{h}\right\|
$$

also holds. Since $\left\|\overline{\boldsymbol{u}}-\boldsymbol{t}_{h}\right\| \rightarrow 0$, then $\left(\boldsymbol{e}_{h}, r_{h}\right) \rightarrow 0$ in $\boldsymbol{H}^{1} \times L^{2}$. By defining $\overline{\boldsymbol{u}}_{h}=\boldsymbol{e}_{h}+\boldsymbol{t}_{h}$, one has

$$
\left\|\overline{\boldsymbol{u}}-\overline{\boldsymbol{u}}_{h}\right\| \leq\left\|\overline{\boldsymbol{u}}-\boldsymbol{t}_{h}\right\|+\left\|\boldsymbol{e}_{h}\right\| \leq\left\|\overline{\boldsymbol{u}}-\boldsymbol{t}_{h}\right\| .
$$

Therefore, we have proved $\boldsymbol{i}$ ). The statement $\boldsymbol{i i}$ ) holds from the definition of $\overline{\boldsymbol{u}}_{h}$.

Let $\boldsymbol{v} \in C_{c}^{\infty}([0, T) \times \Omega)$ be a free divergence function such that $\boldsymbol{v}(T)=0$. We consider $\boldsymbol{v}_{h}^{n}$ the projection (by a discrete Stokes problem) of $\boldsymbol{v}\left(t^{n}\right)$ given by Lemma 29. We define $\boldsymbol{v}_{h, k} \in L^{\infty}\left(0, T ; \boldsymbol{v}_{h}\right)$ as the piecewise constant function taking the value $\boldsymbol{v}_{h}^{n+1}$ on $\left(t_{n}, t_{n+1}\right]$ and $\widetilde{\boldsymbol{v}}_{h, k} \in C^{0}\left([0, T] ; \boldsymbol{V}_{h}\right)$ the corresponding globally continuous piecewise linear function such that $\widetilde{\boldsymbol{v}}_{h, k}\left(t_{n}\right)=\boldsymbol{v}_{h}^{n}$. Then, as $(h, k) \rightarrow 0$, one has

$$
\begin{gathered}
\boldsymbol{v}_{h, k} \rightarrow \boldsymbol{v} \quad \text { in } \quad L^{\infty}\left(0, T ; \boldsymbol{H}_{0}^{1}(\Omega)\right), \\
\widetilde{\boldsymbol{v}}_{h, k} \rightarrow \boldsymbol{v} \quad \text { in } \quad W^{1, \infty}\left(0, T ; \boldsymbol{H}_{0}^{1}(\Omega)\right) .
\end{gathered}
$$

Taking $\overline{\boldsymbol{u}}_{h}=\boldsymbol{v}_{h}^{n+1}$ as a test function in (63), multiplying by $k$, adding over $n$ and using the identity (discrete integration by parts in time)

$$
\begin{aligned}
\sum_{n=0}^{N-1}\left(\rho_{h}^{n+1} \boldsymbol{u}_{h}^{n+1}-\rho_{h}^{n} \boldsymbol{u}_{h}^{n}, \boldsymbol{v}\left(t_{n+1}\right)\right)= & \left(\rho_{h}^{N} \boldsymbol{u}_{h}^{N}, \boldsymbol{v}_{h}^{N}\right) \\
& -\sum_{n=0}^{N-1}\left(\rho_{h}^{n} \boldsymbol{u}_{h}^{n}, \boldsymbol{v}_{h}^{n+1}-\boldsymbol{v}_{h}^{n}\right)-\left(\rho_{0 h} \boldsymbol{u}_{0 h}, \boldsymbol{v}_{h}^{0}\right)
\end{aligned}
$$


and the fact that $\boldsymbol{v}_{h}^{N}=0$ (since $\boldsymbol{v} \in \mathcal{D}([0, T) \times \Omega)$ ), the following statement holds:

$$
\left\{\begin{array}{l}
-\sum_{n=0}^{N-1}\left(\rho_{h}^{n} \boldsymbol{u}_{h}^{n}, \boldsymbol{v}_{h}^{n+1}-\boldsymbol{v}_{h}^{n}\right)-\left(\rho_{0 h} \boldsymbol{u}_{0 h}, \boldsymbol{v}_{h}^{0}\right) \\
+\sum_{n=0}^{N-1} a\left(\left[\rho_{h}^{n+1}\right]_{T}, \boldsymbol{u}_{h}^{n+1}, \boldsymbol{v}_{h}^{n+1}\right)+\sum_{n=0}^{N-1} c\left(\rho_{h}^{n+1} \boldsymbol{u}_{h}^{n}-\lambda \nabla \rho_{h}^{n+1}, \boldsymbol{u}_{h}^{n+1}, \boldsymbol{v}_{h}^{n+1}\right) \\
=k \sum_{n=0}^{N-1}\left(\left[\rho_{h}^{n+1}\right]_{T} \boldsymbol{f}^{n+1}, \boldsymbol{v}_{h}^{n+1}\right)+k \sum_{n=0}^{N-1} \frac{1}{2}\left(\frac{\left[\rho_{h}^{n+1}\right]_{T}-\left[\rho_{h}^{n}\right]_{T}}{k}, \boldsymbol{u}_{h}^{n+1} \cdot \boldsymbol{v}_{h}^{n+1}\right) .
\end{array}\right.
$$

Next, using Definition 17 .

$$
\left\{\begin{array}{l}
-\int_{0}^{T}\left(\widehat{\rho}_{h, k}(t) \widehat{\boldsymbol{u}}_{h, k}(t), \frac{\partial}{\partial t} \widetilde{\boldsymbol{v}}_{h, k}(t)\right) d t-\left(\rho_{0 h} \boldsymbol{u}_{0 h}, \boldsymbol{v}_{h}^{0}\right) \\
+\int_{0}^{T} c\left(\rho_{h, k}(t) \widehat{\boldsymbol{u}}_{h, k}(t)-\lambda \nabla \rho_{h, k}(t), \boldsymbol{u}_{h, k}(t), \boldsymbol{v}_{h, k}(t)\right) d t \\
+\int_{0}^{T} a\left(\rho_{h, k}(t), \boldsymbol{u}_{h, k}(t), \boldsymbol{v}_{h, k}(t)\right) d t \\
=\int_{0}^{T}\left(\rho_{h, k}^{T}(t) \boldsymbol{f}_{k}(t), \boldsymbol{v}_{h, k}(t)\right) d t+\frac{k}{2} \int_{0}^{T}\left(\frac{\partial}{\partial t} \widetilde{\rho}_{h, k}^{T}(t), \boldsymbol{u}_{h, k}(t) \cdot \boldsymbol{v}_{h, k}(t)\right) d t
\end{array}\right.
$$

where we denote $\rho_{h, k}^{T}, \widetilde{\rho}_{h, k}^{T}, \boldsymbol{f}_{k}$ as in the foregoing definitions.

From this weak statement for the discrete momentum system, one can pass to the limit in a standard way thanks to the convergence properties obtained throughout this work. Notice that, to take the limit in the last term, the estimate of Corollary 22 is used.

Then the limit function $(\rho, \boldsymbol{u})$, jointly with an associated pressure $p$ (obtained a posteriori by de Rham's lemma), is the weak solution of the continuous problem. Notice that, thanks to the uniqueness of this weak solution in $2 D$ domains, it is easy to obtain the convergence of the whole sequences. Consequently, the proof of Theorem 5 is concluded.

\section{Pollution model With mass diffusion}

We design a numerical scheme for problem (8), (9)-(10), following the same ideas of the numerical scheme given in Section 2, where we will replace the stabilization term of the momentum system

$$
-\lambda \int_{\Omega} \frac{M+m}{2}\left(\nabla \boldsymbol{u}_{h}^{n+1}\right)^{t}: \nabla \overline{\boldsymbol{u}}_{h} d \boldsymbol{x}
$$

by the term $\lambda m\left(\nabla \cdot \boldsymbol{u}_{h}^{n+1}, \nabla \cdot \overline{\boldsymbol{u}}_{h}\right)$. As well, the density entering into the diffusion term in the momentum system has been truncated. Thus, we arrive at the following numerical scheme:

Given $\left(\boldsymbol{u}_{h}^{n}, p_{h}^{n}, \rho_{h}^{n}\right) \in \boldsymbol{V}_{h} \times M_{h} \times W_{h}$.

(1) Find $\rho_{h}^{n+1} \in W_{h}$ such that for each $\bar{\rho}_{h} \in W_{h}$ :

$$
\left(\frac{\rho_{h}^{n+1}-\rho_{h}^{n}}{k}, \bar{\rho}_{h}\right)+\left(\boldsymbol{u}_{h}^{n} \cdot \nabla \rho_{h}^{n}, \bar{\rho}_{h}\right)+\lambda\left(\nabla \rho_{h}^{n+1}, \nabla \bar{\rho}_{h}\right)=0
$$


(2) Find $\left(\boldsymbol{u}_{h}^{n+1}, p_{h}^{n+1}\right) \in \boldsymbol{V}_{h} \times M_{h}$ such that for each $\left(\overline{\boldsymbol{u}}_{h}, \overline{p_{h}}\right) \in \boldsymbol{V}_{h} \times M_{h}$ :

$$
\begin{gathered}
\left\{\begin{array}{c}
\left(\left[\rho_{h}^{n}\right]_{T} \frac{\boldsymbol{u}_{h}^{n+1}-\boldsymbol{u}_{h}^{n}}{k}, \overline{\boldsymbol{u}}_{h}\right)+\frac{1}{2}\left(\frac{\left[\rho_{h}^{n+1}\right]_{T}-\left[\rho_{h}^{n}\right]_{T}}{k}, \boldsymbol{u}_{h}^{n+1} \cdot \overline{\boldsymbol{u}}_{h}\right)+\widetilde{a}\left(\left[\rho_{h}^{n+1}\right]_{T}, \boldsymbol{u}_{h}^{n+1}, \overline{\boldsymbol{u}}_{h}\right) \\
+c\left(\rho_{h}^{n+1} \boldsymbol{u}_{h}^{n}-\lambda \nabla \rho_{h}^{n+1}, \boldsymbol{u}_{h}^{n+1}, \overline{\boldsymbol{u}}_{h}\right)=\left(\left[\rho_{h}^{n+1}\right]_{T} \boldsymbol{f}^{n+1}, \overline{\boldsymbol{u}}_{h}\right)+\left(p_{h}^{n+1}, \nabla \cdot \overline{\boldsymbol{u}}_{h}\right),
\end{array}\right. \\
\left(\nabla \cdot \boldsymbol{u}_{h}^{n+1}, \bar{p}_{h}\right)=0,
\end{gathered}
$$

where

$$
\widetilde{a}(\rho, \boldsymbol{u}, \boldsymbol{v})=\lambda\left(\rho\left(\nabla \boldsymbol{u}-(\nabla \boldsymbol{u})^{t}\right), \nabla \boldsymbol{v}\right)+\lambda m(\nabla \cdot \boldsymbol{u}, \nabla \cdot \boldsymbol{v})
$$

and $c(\cdot, \cdot, \cdot)$ is defined as in Section 2. Taking into account the equality (87) in (77) and the lower estimate for the truncated density, it follows that

$$
\widetilde{a}\left([\rho]_{T}, \boldsymbol{u}, \boldsymbol{u}\right) \geq \lambda m \int_{\Omega}\left(|\boldsymbol{r o t} \boldsymbol{u}|^{2}+|\nabla \cdot \boldsymbol{u}|^{2}\right) d \boldsymbol{x} .
$$

Observing that $\int_{\Omega}\left(|\operatorname{rot} \boldsymbol{u}|^{2}+|\nabla \cdot \boldsymbol{u}|^{2}\right) d \boldsymbol{x}=\int_{\Omega}|\nabla \boldsymbol{u}|^{2} d \boldsymbol{x}$ for any $\boldsymbol{u} \in \boldsymbol{H}_{0}^{1}(\Omega)$ thanks to the equality (88) (see Appendix B), we arrive at

$$
\widetilde{a}\left([\rho]_{T}, \boldsymbol{u}, \boldsymbol{u}\right) \geq \lambda m\|\boldsymbol{u}\|^{2} \quad \forall \boldsymbol{u} \in \boldsymbol{H}_{0}^{1}(\Omega) .
$$

Accordingly, following the same arguments developed for the model of linear diffusion, one can arrive at exactly the same conclusions. Notice that the passage to the limit in the new diffusion term $\left(\left[\rho_{h, k}\right]_{T}\left(\nabla \boldsymbol{u}_{h, k}-\nabla \boldsymbol{u}_{h, k}^{t}\right), \nabla \boldsymbol{v}_{h, k}\right)$ is controlled thanks to the convergences $\left[\rho_{h, k}\right]_{T} \rightarrow \rho$ in $L^{2}(Q)$-strong (and in $L^{\infty}(\Omega)$-weak $\star$ ), $\nabla \boldsymbol{u}_{h, k} \rightarrow \nabla \boldsymbol{u}$ in $L^{2}(Q)$-weak and $\nabla \boldsymbol{v}_{h, k} \rightarrow \nabla \boldsymbol{v}$ in $L^{2}(Q)$-strong.

\section{A generalization of the pollution MOdel}

The pollution model (8) is a particular case of a general model derived in [3, 4. For this general model, we will define a numerical scheme (using the main ideas of the previous schemes) which will be unconditionally stable, but the convergence remains as an open problem.

Such a model again begins from the compressible model (11) assuming this time the following decomposition:

$$
\rho \boldsymbol{v}=\rho \boldsymbol{u}-\lambda \nabla \Psi(\rho) \quad \text { with } \quad \nabla \cdot \boldsymbol{u}=0 .
$$

Then, imposing $\mu=\lambda$ the compressible model (11) reads ([3, 4):

$$
\left\{\begin{array}{c}
(\rho \boldsymbol{u})_{t}+\nabla \cdot((\rho \boldsymbol{u}-\lambda \nabla \Psi(\rho)) \otimes \boldsymbol{u} \\
-\lambda \boldsymbol{u} \otimes \nabla \Psi(\rho))-\lambda \nabla \cdot(\Psi(\rho) \nabla \boldsymbol{u})+\nabla P=\rho \boldsymbol{f} \text { in } Q, \\
\nabla \cdot \boldsymbol{u}=0 \text { in } Q, \quad \rho_{t}+\nabla \cdot(\rho \boldsymbol{u}-\lambda \nabla \Psi(\rho))=0 \text { in } Q
\end{array}\right.
$$

where

$$
P=q-(\mu+\tilde{\lambda}) \nabla \cdot\left(\boldsymbol{u}-\frac{\lambda}{\rho} \nabla \Psi(\rho)\right)-\lambda \Psi(\rho)_{t}-\lambda^{2}\left(\Psi(\rho) \nabla \cdot\left(\frac{1}{\rho} \nabla \Psi(\rho)\right)+\frac{|\nabla \Psi(\rho)|^{2}}{\rho}\right) .
$$


Now observe that the $\lambda^{2}$-terms which are not canceled are all of potential type (using an auxiliary function $\varphi$ such that $\nabla \varphi(\rho)=\frac{1}{\rho} \nabla \Psi(\rho)$ ) and they are included into the modified pressure $P$.

Note that when $\Psi(\rho)=\rho$ (then $\varphi(\rho)=\log \rho)$, we arrive at the pollution model (8).

The weak definition for the general model (78) remains as follows (now, equality (5) is again used, replacing $\rho$ by $\Psi(\rho))$ :

Definition 30. A pair $(\rho, \boldsymbol{u})$ is called a weak solution of (78), (99)-(10) in $(0, T)$ if it satisfies:

a) $\boldsymbol{u} \in L^{\infty}(0, T ; \boldsymbol{H}) \cap L^{2}(0, T ; \boldsymbol{V})$, $\rho \in L^{\infty}\left(0, T ; L^{2}(\Omega)\right) \cap L^{2}\left(0, T ; H^{1}(\Omega)\right)$, $0<m \leq \rho(\boldsymbol{x}, t) \leq M$, a.e. $(\boldsymbol{x}, t) \in Q$.

b) $\forall \phi \in C^{1}([0, T] ; \boldsymbol{V})$ such that $\phi(T)=0$,

$$
\begin{aligned}
& \int_{0}^{T}\left\{-\left(\boldsymbol{u}, \rho \phi_{t}+(\rho \boldsymbol{u}-\lambda \nabla \Psi(\rho)) \cdot \nabla \phi\right)+\lambda\left(\Psi(\rho)\left(\nabla \boldsymbol{u}-(\nabla \boldsymbol{u})^{t}\right), \nabla \phi\right)\right\} d t \\
& =\int_{0}^{T}(\rho \boldsymbol{f}, \phi) d t+\left(\rho_{0} \boldsymbol{u}_{0}, \phi(0)\right) .
\end{aligned}
$$

c) $\forall \eta \in C^{1}\left([0, T] ; H^{1}(\Omega)\right)$ such that $\eta(T)=0$,

$$
\int_{0}^{T}\left\{-\left(\rho, \eta_{t}\right)-(\rho \boldsymbol{u}, \nabla \eta)+(\lambda \nabla \Psi(\rho), \nabla \eta)\right\} d t=\left(\rho_{0} \boldsymbol{u}_{0}, \eta(0)\right) .
$$

Remark 31. Observe that the density solution for this general model $\rho \in L^{\infty}(0, T$; $\left.L^{2}(\Omega)\right) \cap L^{2}\left(0, T ; H^{1}(\Omega)\right)$; therefore it loses one regularity order in space in comparison with the two earlier models (7) and (8) for which $\rho \in L^{\infty}\left(0, T ; H^{1}(\Omega)\right) \cap$ $L^{2}\left(0, T ; H^{2}(\Omega)\right)$. This regularity implies $\rho_{t} \in L^{2}\left(0, T ; L^{2}(\Omega)\right)$. Now, only $\rho_{t} \in$ $L^{2}\left(0, T ; H^{1}(\Omega)^{\prime}\right)$ holds.

The existence of weak solutions for this general model (178) is established in $([3,4)$.

Theorem 32. Let $\boldsymbol{u}_{0} \in \boldsymbol{H}, \rho_{0} \in H^{1}(\Omega)$ satisfying (11), $\boldsymbol{f} \in L^{2}\left(0, T ; \boldsymbol{L}^{p}(\Omega)\right)$ with $p>1$ and $\Psi \in C^{1}([m, M])$ is a real function such that $0<\alpha \leq \Psi$ and $0<\beta \leq \Psi^{\prime}$ in $[m, M]$. Then, there exists at least a weak solution of (78), (9) -(10) in $(0, T)$.

In order to approximate numerically the general model (78), we propose the following scheme inspired in the foregoing ideas for the models (77) and (8):

Initialization: Let $\left(\boldsymbol{u}_{h}^{0}, \rho_{h}^{0}\right) \in\left(\boldsymbol{V}_{h}, W_{h}\right)$ be suitable approximations of $\left(\boldsymbol{u}_{0}, \rho_{0}\right)$ as $h \rightarrow 0$.

Time step $n+1$ : Given $\left(\boldsymbol{u}_{h}^{n}, \rho_{h}^{n}, \Psi_{h}^{n}\right) \in \boldsymbol{V}_{h} \times W_{h} \times W_{h}$.

(1) Find $\rho_{h}^{n+1} \in W_{h}$ such that for each $\bar{\rho}_{h} \in W_{h}$ :

$$
\left(\frac{\rho_{h}^{n+1}-\rho_{h}^{n}}{k}, \bar{\rho}_{h}\right)+c\left(\boldsymbol{u}_{h}^{n}, \rho_{h}^{n+1}, \bar{\rho}_{h}\right)+\lambda\left(\Psi^{\prime}\left(\left[\rho_{h}^{n}\right]_{T}\right) \nabla \rho_{h}^{n+1}, \nabla \bar{\rho}_{h}\right)=0,
$$


(2) Find $\left(\boldsymbol{u}_{h}^{n+1}, p_{h}^{n+1}\right) \in \boldsymbol{V}_{h} \times M_{h}$ such that for each $\left(\overline{\boldsymbol{u}}_{h}, \overline{p_{h}}\right) \in \boldsymbol{V}_{h} \times M_{h}$ :

(80)

$$
\begin{gathered}
\left\{\begin{array}{c}
\left(\left[\rho_{h}^{n}\right]_{T} \frac{\boldsymbol{u}_{h}^{n+1}-\boldsymbol{u}_{h}^{n}}{k}, \overline{\boldsymbol{u}}_{h}\right)+\frac{1}{2}\left(\frac{\left[\rho_{h}^{n+1}\right]_{T}-\left[\rho_{h}^{n}\right]_{T}}{k}, \boldsymbol{u}_{h}^{n+1} \cdot \overline{\boldsymbol{u}}_{h}\right) \\
+a\left(\Psi\left(\left[\rho_{h}^{n+1}\right]_{T}\right), \boldsymbol{u}_{h}^{n+1}, \overline{\boldsymbol{u}}_{h}\right)+c\left(\rho_{h}^{n+1} \boldsymbol{u}_{h}^{n}-\lambda \Psi^{\prime}\left(\left[\rho_{h}^{n+1}\right]_{T}\right) \nabla \rho_{h}^{n+1}, \boldsymbol{u}_{h}^{n+1}, \overline{\boldsymbol{u}}_{h}\right) \\
=\left(\left[\rho_{h}^{n+1}\right]_{T} \boldsymbol{f}^{n+1}, \overline{\boldsymbol{u}}_{h}\right)+\left(p_{h}^{n+1}, \nabla \cdot \overline{\boldsymbol{u}}_{h}\right), \\
\left(\nabla \cdot \boldsymbol{u}_{h}^{n+1}, \bar{p}_{h}\right)=0,
\end{array}\right.
\end{gathered}
$$

where the trilineal forms $a(\cdot, \cdot, \cdot)$ and $c(\cdot, \cdot, \cdot)$ and the discrete truncation operator $[\cdot]_{T}$ are defined as before.

The convective term for $\rho_{h}^{n+1}$ has been approximated by $c\left(\boldsymbol{u}_{h}^{n}, \rho_{h}^{n+1}, \bar{\rho}_{h}\right)$ instead of $\left(\boldsymbol{u}_{h}^{n} \cdot \nabla \rho_{h}^{n}, \bar{\rho}_{h}\right)$ in order to obtain unconditional stability, since the control of the explicit form introduces constraints between the discretization parameters because the estimates for the density are now satisfied only in weak norms.

In fact, using the techniques developed for the foregoing schemes there are no additional difficulties in obtaining the following unconditional stability estimates:

Lemma 33. Suppose $\boldsymbol{u}_{0} \in \boldsymbol{H}, \rho_{0} \in L^{2}(\Omega)$ satisfying (11), $\boldsymbol{f} \in L^{2}\left(0, T ; \boldsymbol{L}^{p}(\Omega)\right)$ with $p>1$ and $\Psi \in C^{1}([m, M])$ is a real function such that $0<\alpha \leq \Psi$ and $0<\beta \leq \Psi^{\prime}$ in $[m, M]$. Then, the solution of the discrete scheme (79)-(81) satisfies the following estimates:

$$
\begin{aligned}
& \text { i) } \max _{0 \leq n \leq N}\left|\boldsymbol{u}_{h}^{n}\right| \leq C, \quad \text { ii) } k \sum_{n=1}^{N}\left\|\boldsymbol{u}_{h}^{n}\right\|^{2} \leq C, \quad \text { iii) } \sum_{\substack{n=0 \\
N-1}}^{N-1}\left|\boldsymbol{u}_{h}^{n+1}-\boldsymbol{u}_{h}^{n}\right|^{2} \leq C, \\
& \text { iv) } \left.\left.\max _{0 \leq n \leq N}\left|\rho_{h}^{n}\right| \leq C, \quad \boldsymbol{v}\right) k \sum_{n=1}^{N}\left|\nabla \rho_{h}^{n}\right|^{2} \leq C, \quad \boldsymbol{v i}\right) \sum_{n=0}^{N-1}\left|\nabla\left(\rho_{h}^{n+1}-\rho_{h}^{n}\right)\right|^{2} \leq C,
\end{aligned}
$$

with $C>0$ depending only on $\left(\rho_{0}, \boldsymbol{u}_{0}, \boldsymbol{f}\right)$.

For the convergence of the scheme (79)-(81), the main difficulty lies in the compactness argument of the approximate velocity in $\boldsymbol{L}^{2}(Q)$ based on a fractional estimate (see Proposition (27), for which it was essential to control the terms

$$
\frac{k^{2}}{2} \sum_{m=0}^{N-r} \sum_{n=m}^{m-1+r}\left(\frac{\left[\rho_{h}^{n+1}\right]_{T}-\left[\rho_{h}^{n}\right]_{T}}{k}, \boldsymbol{u}_{h}^{n+1} \cdot \boldsymbol{u}_{h}^{m+r}-\boldsymbol{u}_{h}^{m}\right)
$$

and

$$
k^{2} \sum_{m=0}^{N-r} \sum_{n=m}^{m-1+r} c\left(\rho_{h}^{n+1} \boldsymbol{u}_{h}^{n}-\lambda \Psi^{\prime}\left(\left[\rho_{h}^{n+1}\right]_{T}\right) \nabla \rho_{h}^{n+1}, \boldsymbol{u}_{h}^{m+r}-\boldsymbol{u}_{h}^{m}, \boldsymbol{u}_{h}^{n+1}\right)
$$

by $C(r k)^{\gamma}$ with $0<\gamma \leq 1$. Now, because of the loss of regularity of the discrete density with respect to the models (7) and (8), the estimate for the discrete time derivative of the approximate density in $L^{2}(Q)$ changes by an estimate in $L^{2}\left(0, T ; H^{1}(\Omega)^{\prime}\right)$, which is not enough to bound (82) and obviously the weak regularity of the density is not sufficient to bound (83). 


\section{Appendix A. Outline of the proof of Theorem 3}

We only argue in a formal manner. Let us suppose that we have a regular enough solution $(\rho, \boldsymbol{u})$ of (7), (9)-(10).

First, thanks to the maximum principle applied to the parabolic problem for the density (7) ${ }_{c}$ and using (11), we can deduce that

$$
0<m \leq \rho(\boldsymbol{x}, t) \leq M \quad \text { in } Q .
$$

To obtain a priori estimates for the velocity we need to add to the momentum equation (7) a multiplied by $\boldsymbol{v} \in \boldsymbol{V}$ and integrated over $\Omega$, the density equation multiplied by $\frac{1}{2} \boldsymbol{u} \cdot \boldsymbol{v}$ and integrated over $\Omega$, resulting in the variational equality:

$$
\begin{aligned}
& \left(\rho \boldsymbol{u}_{t}, \boldsymbol{v}\right)+(((\rho \boldsymbol{u}-\lambda \nabla \rho) \cdot \nabla) \boldsymbol{u}, \boldsymbol{v})+\left(\mu \nabla \boldsymbol{u}-\lambda \rho(\nabla \boldsymbol{u})^{t}, \nabla \boldsymbol{v}\right) \\
& +\frac{1}{2}\left(\rho_{t}, \boldsymbol{u} \cdot \boldsymbol{v}\right)-\frac{1}{2}(\rho \boldsymbol{u}-\lambda \nabla \rho, \nabla(\boldsymbol{u} \cdot \boldsymbol{v}))=(\rho \boldsymbol{f}, \boldsymbol{v}) .
\end{aligned}
$$

Choosing as a test function $\boldsymbol{v}=\boldsymbol{u}$, we get the following energy relation:

$$
\frac{1}{2} \frac{d}{d t} \int_{\Omega} \rho|\boldsymbol{u}|^{2} d \boldsymbol{x}+\mu\|\boldsymbol{u}\|^{2}=\lambda \int_{\Omega} \rho(\nabla \boldsymbol{u})^{t}: \nabla \boldsymbol{u} d \boldsymbol{x}+(\rho \boldsymbol{f}, \boldsymbol{u}) .
$$

Making use of the equality

$$
\int_{\Omega}(\nabla \boldsymbol{v})^{t}: \nabla \boldsymbol{v} d \boldsymbol{x}=0, \quad \forall \boldsymbol{v} \in \boldsymbol{v}
$$

one can rewrite the first term on the right-hand side of (84) as

$$
\int_{\Omega} \rho(\nabla \boldsymbol{u})^{t}: \nabla \boldsymbol{u} d \boldsymbol{x}=\int_{\Omega}\left(\rho-\frac{M+m}{2}\right)(\nabla \boldsymbol{u})^{t}: \nabla \boldsymbol{u} d \boldsymbol{x} .
$$

Since $m \leq \rho \leq M$, the inequality $|\rho-(M+m) / 2| \leq(M-m) / 2$ holds almost everywhere in $Q$; therefore

$$
\lambda \int_{\Omega} \rho(\nabla \boldsymbol{u})^{t}: \nabla \boldsymbol{u} d \boldsymbol{x} \leq \lambda \frac{M-m}{2}\|\boldsymbol{u}\|^{2} .
$$

Imposing the restriction on the coefficients, $\lambda<2 \mu(M-m)^{-1}$, this gives $\mu-\frac{\lambda}{2}(M-$ $m)=\mu_{1} / 2>0$. Then, from (84), (85) and the upper bound for the density,

$$
\frac{d}{d t} \int_{\Omega} \rho|\boldsymbol{u}|^{2} d \boldsymbol{x}+\mu_{1}\|\boldsymbol{u}\|^{2} \leq 2(\rho \boldsymbol{f}, \boldsymbol{u}) \leq \varepsilon\|\boldsymbol{u}\|^{2}+C_{\varepsilon}\|\boldsymbol{f}\|_{L^{p}(\Omega)}^{2}
$$

with $p>1$. Therefore, applying the lower bound of the density and integrating (86) over $(0, t) \forall t \leq T$, we arrive at the estimate

$$
\max _{0 \leq t \leq T}|\boldsymbol{u}(t)|^{2}+\int_{0}^{T}\|\boldsymbol{u}(t)\|^{2} d t \leq C .
$$

Multiplying the density equation (17) $c$ by $-\Delta \rho$, bounding the convective term thanks to the $2 D$ interpolation inequalities

$$
\|\boldsymbol{u}\|_{L^{4}(\Omega)} \leq C|\boldsymbol{u}|^{1 / 2}|\nabla \boldsymbol{u}|^{1 / 2} \quad \text { and } \quad\|\nabla \rho\|_{L^{4}(\Omega)} \leq C|\nabla \rho|^{1 / 2}|\Delta \rho|^{1 / 2}
$$

(which are a consequence of Gagliardo-Nirenberg's inequality and the equivalent norms $\|\boldsymbol{u}\|_{H^{1}(\Omega)}$ and $|\nabla \boldsymbol{u}|_{L^{2}(\Omega)}$, and $\|\nabla \rho\|_{H^{1}(\Omega)}$ and $\left.|\Delta \rho|\right)$, we arrive at

$$
\frac{1}{2} \frac{d}{d t}|\nabla \rho|^{2}+\lambda|\Delta \rho|^{2} \leq C\|\boldsymbol{u}\|_{L^{4}}\|\nabla \rho\|_{L^{4}}|\Delta \rho| \leq \varepsilon|\Delta \rho|^{2}+C|\boldsymbol{u}|^{2}|\nabla \boldsymbol{u}|^{2}|\nabla \rho|^{2} .
$$


Therefore, from Gronwall's Lemma, we get the estimate

$$
\max _{0 \leq t \leq T}|\nabla \rho(t)|^{2}+\lambda \int_{0}^{T}|\Delta \rho(t)|^{2} d t \leq C .
$$

Through a quite technical argument (1]), one arrives at the following estimate of the "fractional in time derivative"

$$
\int_{0}^{T-\delta}|\boldsymbol{u}(t+\delta)-\boldsymbol{u}(t)|^{2} d t \leq C \delta^{1 / 2}, \quad \forall \delta \in(0, T)
$$

This estimate implies ([16]) compactness for the velocity $\boldsymbol{u}$ in $L^{2}\left(0, T ; \boldsymbol{L}^{2}(\Omega)\right)$. From here, it is rather standard to obtain the existence of weak solutions of (1]), using for instance the Faedo-Galerkin method.

\section{Appendix B. Sketch of the Proof of Theorem 4}

Suppose that we have $(\boldsymbol{u}, \rho, q)$ a sufficiently regular solution of (8), (9)-(10). From the maximum principle of the parabolic density equation $(8)_{c}$ and since $0<$ $m \leq \rho_{0}(x) \leq M<+\infty$, one gets $0<m \leq \rho(x, t) \leq M<+\infty$ in $Q$.

Multiplying the momentum system (8) $)_{a}$ by $\boldsymbol{u}$, the density equation (8) $)_{c}$ by $\frac{1}{2} \boldsymbol{u} \cdot \boldsymbol{u}$, integrating over $\Omega$ and using the so-called vorticity tensor $W=\nabla \boldsymbol{u}-\nabla \boldsymbol{u}^{t}$, we arrive at

$$
\int_{\Omega} \rho\left(|\nabla \boldsymbol{u}|^{2}-(\nabla \boldsymbol{u})^{t}: \nabla \boldsymbol{u}\right) d \boldsymbol{x}=\int_{\Omega} \rho W: \nabla \boldsymbol{u} d \boldsymbol{x}=\int_{\Omega} \rho|\operatorname{rot} \boldsymbol{u}|^{2} d \boldsymbol{x} .
$$

Since $W=\operatorname{rot} u\left(\begin{array}{cc}0 & -1 \\ 1 & 0\end{array}\right)$, one has

$$
\frac{d}{d t} \int_{\Omega} \rho|\boldsymbol{u}|^{2} d \boldsymbol{x}+2 \lambda \int_{\Omega} \rho|\operatorname{rot} \boldsymbol{u}|^{2} d \boldsymbol{x}=2 \int_{\Omega} \rho \boldsymbol{f} \cdot \boldsymbol{u} d \boldsymbol{x} .
$$

Using $\Delta=\nabla \operatorname{div}-$ rot rot, one has

$$
\int_{\Omega}|\nabla \boldsymbol{u}|^{2} d \boldsymbol{x}=\int_{\Omega}\left(|\operatorname{rot} \boldsymbol{u}|^{2}+|\nabla \cdot \boldsymbol{u}|^{2}\right) d \boldsymbol{x}
$$

hence since $\nabla \cdot \boldsymbol{u}=0$, we get

$$
\int_{\Omega}|\nabla \boldsymbol{u}|^{2} d \boldsymbol{x}=\int_{\Omega}|\operatorname{rot} \boldsymbol{u}|^{2} d \boldsymbol{x}
$$

Next, applying the lower bound for the density to $\int_{\Omega} \rho|\operatorname{rot} \boldsymbol{u}|^{2} d \boldsymbol{x} \geq m \int_{\Omega}|\boldsymbol{\operatorname { r o t }} \boldsymbol{u}|^{2} d \boldsymbol{x}$ and the identity (89), one arrives at

$$
\frac{d}{d t} \int_{\Omega} \rho|\boldsymbol{u}|^{2} d \boldsymbol{x}+2 \lambda m \int_{\Omega}|\nabla \boldsymbol{u}|^{2} d \boldsymbol{x} \leq 2(\rho \boldsymbol{f}, \boldsymbol{u}) \leq \varepsilon\|\boldsymbol{u}\|^{2}+C_{\varepsilon}\|\boldsymbol{f}\|_{L^{p}(\Omega)}^{2}
$$

where $p>1$. Therefore, integrating (90) over $(0, t) \forall t \leq T$ and applying the lower estimate for the density to the first term, one arrives at

$$
\max _{0 \leq t \leq T}|\boldsymbol{u}(t)|^{2}+\int_{0}^{T}\|\boldsymbol{u}(t)\|^{2} d t \leq C .
$$

The bounds in $L^{\infty}\left(H^{1}\right) \cap L^{2}\left(H^{2}\right)$ for the density are obtained as in Theorem 3 , 


\section{REFERENCES}

1. S. N. Antontsev, A. V. Kazhikhov, V. N. Monakhov. Boundary value problems in mechanics of nonhomogeneous fluids, vol. 22 of Studies in Mathematics and its Applications, North-Holland Publishing Co., Amsterdam, 1990. MR.1035212 (91d:76018)

2. H. BEIRÃo DA VEIGA. Diffusion on viscous fluids. Existence and asymptotic properties of solutions. Ann, Sc. Norm. Sup. Pisa, 10 (1983), 341-355. MR.728440 (86c:76051)

3. D. Bresch, E. H. Essoufi, M. Sy. De nouveaux systèmes de type Kazhikhov-Smagulov: modèles de propagation de polluants et de combustion à faible nombre de Mach, C. R. Acad. Sci. Paris, 335, Série I, (2002), 973-978. MR1952560 (2003m:35196)

4. D. Bresch, E. H. Essoufi, M. Sy. Effects of density dependent viscosities on multiphasic incompressible fluid models, J. Math. Fluid Mech., DOI 10.1007/s00021-005-0204-4.

5. P. G. Ciarlet. The finite element method for elliptic problems, Amsterdam, North-Holland, 1987. MR0520174 (58:25001)

6. J. Étienne, E. J. Hopfinger, P. Saramito. Numerical simulations of high density ratio lock-exchange flows. Phys. Fluids 17, 036601 (2005).

7. J. Étienne, P. Saramito. A priori error estimates of the Lagrange-Galerkin method for Kazhikhov-Smagulov type systems, C. R. Math. Acad. Sci. Paris 341 (2005), no. 12, 769-774. MR 2188875 (2006f:76018)

8. V. Girault, P.A. Raviart, Finite element methods for Navier-Stokes equations: Theory and algorithms, Berlin, Springer-Verlag, 1986. MR851383 (88b:65129)

9. V. Girault, F. Guillén-GonzÁlez. Mixed formulation, approximation and decoupling algorithm for a nematic liquid crystals model. In preparation.

10. F. Guillén-González, P. Damázio, M.A. Rojas-Medar. Approximation by an iterative method for regular solutions for incompressible fluids with mass diffusion. J. Math. Anal. Appl. 326 (2007) 468-487. MR2277796

11. F. Guillén-GonzÁlez, M. SY. An iterative method for mass diffusion model with density dependent viscosity. Submitted.

12. A. Kazhikhov, Sh. Smagulov. The correctness of boundary value problems in a diffusion model of an inhomogeneous fluid. Sov. Phys. Dokl., 22, (1977), No. 1, 249-252.

13. R. SALVI. On the existence of weak solutions of boundary value problems in a diffusion model for an inhomogeneous liquid in regions with moving boundaries, Portugaliae Math. 43 (1986), 213-233. MR886668 (88h:35096)

14. P. SECCHI. On the motion of viscous fluids in the presence of diffusion. Siam J. Math. Anal. 19 (1988), 22-31. MR.924542 (89j:35109)

15. P. SECCHI. On the initial value problem for the equations of motion of viscous incompressible fluids in the presence of diffusion. Bollettino U.M.I., 6 1-B, 1982, 1117-1130. MR683497 (84b:35104)

16. J. Simon. Compact sets in the Space $L^{p}(0, T ; B)$, Ann. Mat. Pura Appl., 146 (1987), 65-97. MR 916688 (89c:46055)

17. R. Temam. Navier-Stokes equations. Theory and numerical analysis, North-Holland Publishing Co., Amsterdam, 1977. MR0609732 (58:29439)

Departamento E.D.A.N., University of Sevilla, Aptdo. 1160, 41080 Sevilla, Spain

E-mail address: guillen@us.es

Departamento E.D.A.N., University of Sevilla, Aptdo. 1160, 41080 Sevilla, Spain

E-mail address: juanvi@us.es 